\title{
A proof of the two-dimensional Markus-Yamabe Stability Conjecture and a generalization
}

\author{
by RoBert FEßLer (Basel)
}

\begin{abstract}
The following problem of Markus and Yamabe is answered affirmatively: Let $f$ be a local diffeomorphism of the euclidean plane whose jacobian matrix has negative trace everywhere. If $f(0)=0$, is it true that 0 is a global attractor of the ODE $d x / d t=$ $f(x)$ ? An old result of Olech states that this is equivalent to the question if such an $f$ is injective. Here the problem is treated in the latter form by means of an investigation of the behaviour of $f$ near infinity.
\end{abstract}

1. Introduction. In this work we solve $\left({ }^{1}\right)$ the following problem which is known as the two-dimensional Global Asymptotic Stability Jacobian Conjecture or Markus-Yamabe Stability Conjecture.

Problem 1. Let $f \in C^{1}\left(\mathbb{R}^{2}, \mathbb{R}^{2}\right)$ be such that:

1. $\operatorname{det} D f(x)>0$ for all $x \in \mathbb{R}^{2}$.

2. $\operatorname{tr} D f(x)<0$ for all $x \in \mathbb{R}^{2}$.

3. $f(0)=0$.

Here $D f(x)$ denotes the Jacobian matrix, det the determinant and tr the trace. Is it true that under the conditions 1-3 every solution of

$$
\dot{x}(t)=f(x(t))
$$

approaches 0 as $t \rightarrow \infty$ ?

This problem and its $n$-dimensional reformulation go back to Markus and Yamabe $[\mathrm{MY}]$ in 1960.

1991 Mathematics Subject Classification: 34D05, 34D45, 57R30, 57R40, 57R42.

Key words and phrases: Markus-Yamabe conjecture, asymptotic behaviour of solutions of ODE's, immersions, embeddings, injectivity of mappings, curve lifting, foliations.

$\left({ }^{1}\right)$ I acknowledge that Carlos Gutierrez has also solved this problem independently. Both he and the present author presented their proofs $[\mathrm{Gu}]$, [Fe] on the conference about Recent Results on the Global Asymptotic Stability Jacobian Conjecture, Università di Trento, Povo, Italy, September 1993. 
In the two-dimensional case several authors achieved an affirmative answer to this problem under various additional assumptions. Krasovskiu $[\mathrm{Kr}]$ solved a related problem with a certain growth condition on $f$. Markus and Yamabe $[\mathrm{MY}]$ treated the case when one of the partial derivatives of $f$ vanishes identically on $\mathbb{R}^{2}$. Hartman [Ha] used the stronger hypothesis that the symmetric part of $D f(x)$ is negative definite everywhere. His result is also valid in higher dimensions. Olech [Ol] solved the problem affirmatively if $|f|$ is bounded from below in some neighbourhood of infinity. A generalization to higher dimensions can be found in Hartman and Olech [HO]. In 1988 Meisters and Olech [MO] proved the conjecture for polynomial maps. The attention of the author was attracted to the problem by an article of Gasull, Llibre and Sotomayor [GLS] where the relation of this conjecture to several other problems was investigated. Barabanov [Ba] showed that this conjecture is false if $n \geq 4$.

Olech [Ol] proved in 1963 that Problem 1 is equivalent to

Problem 2. Let $f \in C^{1}\left(\mathbb{R}^{2}, \mathbb{R}^{2}\right)$ be such that:

1. $\operatorname{det} D f(x)>0$ for all $x \in \mathbb{R}^{2}$.

2. $\operatorname{tr} D f(x)<0$ for all $x \in \mathbb{R}^{2}$.

Is it true that $f$ is injective?

This gives the key to our solution. Our Theorem 1 is an affirmative answer to Problem 2. Actually, it is even more general: The hypotheses of Problem 2 are equivalent to assuming that the eigenvalues of $D f(x)$ have negative real parts for all $x \in \mathbb{R}^{2}$. Therefore hypothesis 2 of our Theorem 1 is weaker than hypothesis 2 of Problem 2. Furthermore, we only need it in a neighbourhood of infinity.

\section{The solution of the problem}

Theorem 1. Let $f \in C^{1}\left(\mathbb{R}^{2}, \mathbb{R}^{2}\right)$ be such that:

1. $\operatorname{det} D f(x)>0$ for all $x \in \mathbb{R}^{2}$ (i.e. $f$ is a local diffeomorphism).

2. There is a compact set $K \subset \mathbb{R}^{2}$ such that $D f(x) v \neq \lambda v$ for all $x \in \mathbb{R}^{2} \backslash K, v \in \mathbb{R}^{2} \backslash\{0\}$ and $\left.\lambda \in\right] 0, \infty[$ (i.e. $D f(x)$ has no real positive eigenvalues for any $x$ in some neighbourhood of infinity).

Then $f$ is injective.

Proof. The proof will be given using several definitions and lemmata. We assume throughout that $f$ is not injective. Only using hypothesis 1 of Theorem 1 we thus arrive at Lemma 10. Since this is a general result about non-injective self-immersions of the plane we restate it as Theorem 2. At this point we also need a general result about certain curves in the plane 
which is given in Theorem 3. Combining both we will finally arrive at a contradiction to hypothesis 2 of Theorem 1 .

Thus, if $f$ is not injective we will find $x_{0}, x_{1} \in \mathbb{R}^{2}, x_{0} \neq x_{1}$, such that $f\left(x_{0}\right)=f\left(x_{1}\right)$. Without loss of generality we may assume that $f\left(x_{0}\right)=$ $f\left(x_{1}\right)=0$.

Definition 1. We define $C$ to be the set of all curves $\alpha \in C^{1}\left([0,1], \mathbb{R}^{2}\right)$ such that:

(i) $\forall s \in[0,1]: \dot{\alpha}(s) \neq 0$.

(ii) $\alpha(0)=x_{0}, \alpha(1)=x_{1}$.

(iii) $\alpha$ is injective.

(iv) $\alpha(] 0,1[) \cap f^{-1}(0)=\emptyset$.

Lemma 1. $C \neq \emptyset$.

Proof. Let $\alpha_{l}(s):=(1-s) x_{0}+s x_{1}$ be the straight line segment from $x_{0}$ to $x_{1}$. Then $\alpha$ obviously has all properties in order to be contained in $C$ except for (iv). Since $f$ is a local homeomorphism, the set $f^{-1}(0)$ is discrete. Therefore we can slightly modify $\alpha_{l}$ near the (finitely many) points of $\alpha_{l}(] 0,1[) \cap f^{-1}(0)$ so that this set becomes empty (see (iv)). Of course, this can be done in such a way that the other properties required for a curve to be in $C$ remain valid.

Definition\&Lemma 1. 1. Every curve $\beta \in C^{0}\left(I, \mathbb{R}^{2} \backslash\{0\}\right)(I=][a, b][$ being an arbitrary interval) induces an angle function

$$
\angle \beta \in C^{0}(I, \mathbb{R}), \quad \angle \beta(s):=\arg \beta_{\mathbb{C}}(s) .
$$

Here $\beta_{\mathbb{C}} \in C^{0}(I, \mathbb{C} \backslash\{0\})$ denotes the curve $\beta$ composed with the canonical identification of $\mathbb{R}^{2}$ with the complex plane $\mathbb{C}$, and arg denotes a continuous branch of the complex argument function. (Later we will use the fact that $\arg z=\operatorname{Im} \ln z$ on every simply connected area of $\mathbb{C} \backslash\{0\}$ with an appropriately chosen branch of the logarithm $\mathrm{ln}$.)

If $0 \in I$ we choose $\arg \beta_{\mathbb{C}}(0) \in[0,2 \pi[$ unless otherwise stated. Moreover, if $\beta \in C^{1}\left(I, \mathbb{R}^{2} \backslash\{0\}\right)$ then also $\angle \beta \in C^{1}(I, \mathbb{R})$.

2. If $\beta \in C^{1}\left(\left[a, b\left[, \mathbb{R}^{2}\right)\right.\right.$ (or $\left.\left.\beta \in C^{1}(] a, b\right], \mathbb{R}^{2}\right)$, resp.) is such that $0 \notin$ $\beta(] a, b[)$ and

$$
\beta(a)=0, \dot{\beta}(a) \neq 0 \quad(\text { or } \beta(b)=0, \dot{\beta}(b) \neq 0, \text { resp. })
$$

then

$$
\begin{gathered}
\lim _{s \searrow a} \angle \beta(s)=L \dot{\beta}(a)+2 \pi k, \quad k \in \mathbb{Z} \\
\text { (or } \lim _{s \nearrow b} \angle \beta(s)=\angle \dot{\beta}(b)+\pi+2 \pi k, k \in \mathbb{Z}, \text { resp.) }
\end{gathered}
$$

Therefore we may extend the function $\angle \beta \in C^{0}(] a, b[, \mathbb{R})$ continuously to $[a, b[$ (or $] a, b]$, resp.) in this case. 
Proof. 1. Since $0 \notin \beta(I), \arg \beta_{\mathbb{C}}$ is defined on $I$. The definition of $\angle \beta$ shows that $(\angle \beta)^{\circ}=\operatorname{Im} \dot{\beta} / \beta$. Therefore, $\beta$ being $C^{1}$ implies $\angle \beta$ being $C^{1}$.

2. Since $\beta$ is differentiable at $a$ and $\beta(a)=0$ we know that $\beta(s)=$ $\dot{\beta}(a)(s-a)+\varphi(s-a)$, with a $\varphi \in o($ id $)$. Hence

$$
\begin{aligned}
\angle \beta(s) \bmod 2 \pi \beta_{\mathbb{C}} & =\operatorname{Im} \ln \beta_{\mathbb{C}}(s) \\
& =\operatorname{Im} \ln (s-a)\left(\dot{\beta}_{\mathbb{C}}(a)+\varphi_{\mathbb{C}}(s-a) /(s-a)\right) \\
& =\operatorname{Im}\left(\ln (s-a)+\ln \left(\dot{\beta}_{\mathbb{C}}(a)+\varphi_{\mathbb{C}}(s-a) /(s-a)\right)\right) \\
& =\operatorname{Im} \ln \left(\dot{\beta}_{\mathbb{C}}(a)+\varphi_{\mathbb{C}}(s-a) /(s-a)\right) \\
& \rightarrow \operatorname{Im} \ln \dot{\beta}_{\mathbb{C}}(a)=\angle \dot{\beta}(a) \bmod 2 \pi \quad \text { as } s \searrow a .
\end{aligned}
$$

The proof for $s \nearrow b$ is analogous. However, since $s-b<0$ in this case we obtain a summand $\pi$ added.

Definition\&Lemma 2. 1. Every curve $\alpha \in C$ induces functions $\angle \dot{\alpha}$, $\angle f \circ \alpha, \angle(f \circ \alpha)^{\cdot} \in C^{0}([0,1], \mathbb{R})$. Moreover, $\angle f \circ \alpha \in C^{1}(] 0,1[, \mathbb{R}]$. by

2. For every curve $\alpha \in C$ we also define the function $\Theta_{\alpha} \in C^{0}([0,1], \mathbb{R})$

$$
\Theta_{\alpha}(s):=\angle(f \circ \alpha)^{\cdot}(s)-\angle f \circ \alpha(s)
$$

and observe that $\Theta_{\alpha}(0) \bmod 2 \pi=0$ and $\Theta_{\alpha}(1) \bmod 2 \pi=\pi$.

3 . We will call the curve $\beta \in C^{0}\left([a, b], \mathbb{R}^{2}\right)$ piecewise regular (p.w. regular) if it is locally injective and if there exist $s_{i}, i=1, \ldots, n$, such that $a=s_{1}<$ $\ldots<s_{n}=b$ and that $\beta_{i}:=\beta \mid\left[s_{i}, s_{i+1}\right]$ is regular for all $i=1, \ldots, n-1$. $\beta_{i}$ being regular means that $\beta_{i}$ is continuously differentiable (at $s_{i}$ and $s_{i+1}$ we consider one-sided differentials) and that $\dot{\beta}_{i}(s) \neq 0$ for all $s \in\left[s_{i}, s_{i+1}\right]$.

For such curves we may also define a unique tangent angle function $L \dot{\beta}$ as follows: If we assume that $s \in\left[s_{i}, s_{i+1}\right]$ then

$$
\angle \dot{\beta}(s):=\angle \dot{\beta}_{i}(s)-\angle \dot{\beta}_{i}\left(s_{i}\right)+\sum_{k=1}^{i-1} \angle \dot{\beta}_{k}\left(s_{k+1}\right)-\angle \dot{\beta}_{k}\left(s_{k}\right)+\delta_{k+1},
$$

where $\delta_{k+1}$ denotes the "tangent angle jump" in the edge of $\beta$ at $s_{k+1}$. We will define it in the following way: Let $\Delta_{k} \beta_{k-1}(h):=\beta_{k-1}\left(s_{k}\right)-\beta_{k-1}\left(s_{k}-h\right)$ and $\Delta_{k} \beta_{k}(h):=\beta_{k}\left(s_{k}+h\right)-\beta_{k}\left(s_{k}\right)$. Since $\dot{\beta}_{k-1}\left(s_{k}\right), \dot{\beta}_{k}\left(s_{k}\right) \neq 0$ there are unique functions $h_{0}, h_{1} \in C^{0}\left(\left[0, \varepsilon\left[,\left[0, \infty[)\right.\right.\right.\right.$ with $h_{0}(0)=h_{1}(0)=0$ such that

$$
\left\|\Delta_{k} \beta_{k-1}\left(h_{0}(r)\right)\right\|_{2}=r, \quad\left\|\Delta_{k} \beta_{k}\left(h_{1}(r)\right)\right\|_{2}=r
$$

for all $r \in\left[0, \varepsilon\left[\right.\right.$ (implicit function theorem). Let $\left.\left.\varphi_{|\pi|} \in\right]-\pi, \pi\right]$ denote the unique angle with $\varphi_{|\pi|}=\varphi \bmod 2 \pi$. Since $\beta$ is locally injective, the angle

$$
\widehat{\delta}_{k}(r):=\left(\angle \Delta_{k} \beta_{k}\left(h_{1}(r)\right)-\angle \Delta_{k} \beta_{k-1}\left(h_{0}(r)\right)\right)_{|\pi|}
$$

never equals $\pi$ for small $r>0$. Therefore, $\widehat{\delta}_{k}$ is continuous for such $r$ and 
$\lim _{r \rightarrow 0} \widehat{\delta}_{k}(r) \in[-\pi, \pi]$ exists. Thus, we may finally define

$$
\delta_{k}:=\lim _{r \rightarrow 0} \widehat{\delta}(r) \in[-\pi, \pi] .
$$

Proof. 1. $\alpha \in C \Rightarrow \dot{\alpha},(f \circ \alpha)$ ' are continuous and never 0 (see Definition 1$) \Rightarrow L \dot{\alpha}, \angle(f \circ \alpha)^{\cdot}$ are defined and continuous (see Definition\&Lemma 1.1).

$\alpha \in C \Rightarrow f \circ \alpha \in C^{1}\left([0,1], \mathbb{R}^{2}\right), 0 \notin f \circ \alpha(] 0,1[)$,

$f \circ \alpha(0)=f \circ \alpha(1)=0 \Rightarrow \angle f \circ \alpha$ is defined on $[0,1]$ and is continuously differentiable (see Definition\&Lemma 1).

2. This is obvious from Definition\&Lemma 1.2.

Lemma 2. If $\left.\Theta_{\alpha}(s) \bmod 2 \pi \in\right] 0, \pi[(\in] \pi, 2 \pi[$, resp. $)$ for all $s \in] s_{1}, s_{2}[$ then $\angle f \circ \alpha$ is strictly increasing (strictly decreasing, resp.) on $\left[s_{1}, s_{2}\right]$.

P r o of. We conclude from our hypothesis that $0,1 \notin] s_{1}, s_{2}\left[\right.$ since $\Theta_{\alpha}(s)$ $\bmod 2 \pi \in\{0, \pi\}$ if $s \in\{0,1\}$. Therefore, using our definitions we may calculate:

$$
\begin{aligned}
(\angle f \circ \alpha)^{\cdot} & =\operatorname{Im}(f \circ \alpha)_{\mathbb{C}} /(f \circ \alpha)_{\mathbb{C}} \\
& =\operatorname{Im}\left|(f \circ \alpha)_{\mathbb{C}}\right| e^{i L(f \circ \alpha)^{\cdot}} /(f \circ \alpha)_{\mathbb{C}} \\
& \left.=\operatorname{Im}\left(\left|(f \circ \alpha)_{\mathbb{C}}\right| /(f \circ \alpha)\right)_{\mathbb{C}}\right) e^{i L(f \circ \alpha)+i \Theta_{\alpha}} \\
& =\operatorname{Im} \frac{\left|(f \circ \alpha)_{\mathbb{C}}\right| \overline{(f \circ \alpha)_{\mathbb{C}}}}{\left|(f \circ \alpha)_{\mathbb{C}}\right|^{2}} \cdot \frac{(f \circ \alpha)_{\mathbb{C}}}{\left|(f \circ \alpha)_{\mathbb{C}}\right|} e^{i \Theta_{\alpha}}=\frac{\left|(f \circ \alpha)_{\mathbb{C}}\right|}{\left|(f \circ \alpha)_{\mathbb{C}}\right|} \sin \Theta_{\alpha} .
\end{aligned}
$$

Applying Rolle's theorem yields the assertion.

Definition 2 (See Figure 1). 1. For every $\alpha \in C$ we define the family of rays

$$
\Gamma_{\alpha} \in C^{1}(] 0,1\left[\times\left[0, \infty\left[, \mathbb{R}^{2}\right) \quad \text { by } \quad \Gamma_{\alpha}(s, t):=t \cdot f \circ \alpha(s) .\right.\right.
$$

(Notice that for every $s \in] 0,1\left[\right.$ the curve $\Gamma_{\alpha}(s, \cdot)$ is the straight ray emanating from 0 and passing through $f \circ \alpha(s) \neq 0$ (Definition 1(iv)).)

2. Moreover, we need the $f$-induced lift of the family $\Gamma_{\alpha}$, denoted by $\Gamma_{\alpha}^{f}: \Omega_{\alpha} \rightarrow \mathbb{R}^{2}$ with $\left.\Omega_{\alpha} \subset\right] 0,1[\times[0, \infty[$. We define it by lifting every ray $\Gamma_{\alpha}(s, \cdot)$ of the family separately, i.e.

$$
\Gamma_{\alpha}^{f}(s, \cdot):=\left(\Gamma_{\alpha}(s, \cdot)\right)^{f}: \Omega_{\alpha}(s) \rightarrow \mathbb{R}^{2},
$$

where we choose the unique lift such that

$$
\Gamma_{\alpha}^{f}(s, 1):=\alpha(s) \in f^{-1}\left(\Gamma_{\alpha}(s, 1)\right) .
$$

It is defined on a maximal open interval of existence $\Omega_{\alpha}(s) \subset[0, \infty[$ with $1 \epsilon$ $\Omega_{\alpha}(s)$. Then $\Omega_{\alpha}=\bigcup_{s \in] 0,1[}\{s\} \times \Omega_{\alpha}(s)$. Note that $\Omega_{\alpha}$ is open in $] 0,1[\times[0, \infty[$. 

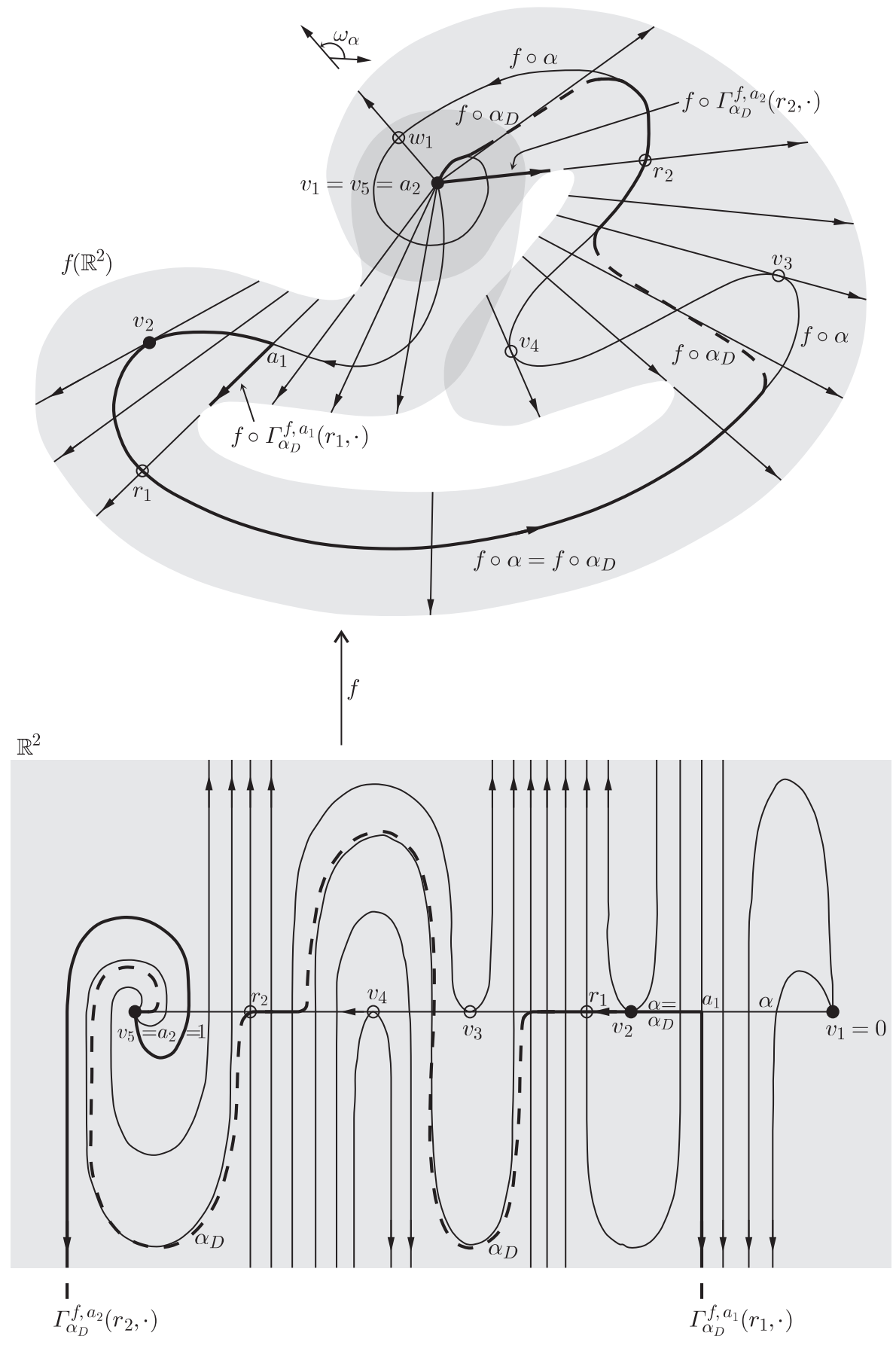

Fig. 1 
We will often use the following simple observation: If $\Gamma_{\alpha}^{f}\left(s_{1}, t_{1}\right)=$ $\Gamma_{\alpha}^{f}\left(s_{2}, t_{2}\right)$ (or $\Gamma_{\alpha}\left(s_{1}, t_{1}\right)=\Gamma_{\alpha}\left(s_{2}, t_{2}\right)$, resp.) and $t_{1}, t_{2} \neq 0$ then

$$
\operatorname{im} \Gamma_{\alpha}^{f}\left(s_{1}, \cdot\right)=\operatorname{im} \Gamma_{\alpha}^{f}\left(s_{2}, \cdot\right) \quad\left(\operatorname{im} \Gamma_{\alpha}\left(s_{1}, \cdot\right)=\operatorname{im} \Gamma_{\alpha}\left(s_{2}, \cdot\right), \text { resp. }\right) .
$$

(Thus, the sets $\Gamma_{\alpha}^{f}\left(\{s\} \times\left(\Omega_{\alpha}(s) \backslash\{0\}\right)\right)$ and $\Gamma_{\alpha}(\{s\} \times] 0, \infty[)$, resp. may be considered as leaves of a foliation on some open subset of $\mathbb{R}^{2} \backslash f^{-1}(0)$ or $\mathbb{R}^{2} \backslash\{0\}$, resp.)

3. $\Gamma_{\alpha}^{f} \in C^{1}\left(\Omega_{\alpha}, \mathbb{R}^{2}\right)$ and for every $\left.s \in\right] 0,1[$ we may define the maps $\angle \Gamma_{\alpha}(s, \cdot) \in C^{1}\left(\left[0, \infty[, \mathbb{R}), \angle \dot{\Gamma}_{\alpha}(s, \cdot) \in C^{0}\left(\left[0, \infty[, \mathbb{R})\right.\right.\right.\right.$ and $\angle\left(\Gamma_{\alpha}^{f}\right) \cdot(s, \cdot) \in$ $C^{0}\left(\Omega_{\alpha}(s), \mathbb{R}\right)$ according to Definition\&Lemma 1.

4. $\angle \dot{\Gamma}_{\alpha}(s, t)=\angle \Gamma_{\alpha}(s, t)=\angle f \circ \alpha(s) \bmod 2 \pi$ for $\left.s \in\right] 0,1[$ and $t \in] 0, \infty[$.

Now we aim at a modification of our curve $\alpha$ (see Definition\&Lemma 6 ) such that it has at most two intersections with every lifted ray $\Gamma_{\alpha}^{f}(\bar{s}, \cdot)$ (tangencies are counted twice). To this end we use a finite iteration of the modification step of Definition\&Lemma 5. In order to prove the finiteness of this iteration we need the set $V_{\alpha} \cup W_{\alpha}$ of exceptional curve parameters (Definition\&Lemma 3). It is related to the number of intersections of $\alpha$ with $\operatorname{im} \Gamma_{\alpha}^{f}(\bar{s}, \cdot)$ (Lemma 3, Definition\&Lemma 4). We show that every modification step strictly decreases the number of elements in $V_{\alpha} \cup W_{\alpha}$.

Definition\&Lemma 3 (See Figure 1). 1. For every curve $\alpha \in C$ we define

$$
V_{\alpha}:=\left\{s \in[0,1] \mid \Theta_{\alpha}(s) \bmod \pi=0\right\},
$$

i.e. $V_{\alpha}$ is the set of all $s$ where $f \circ \alpha$ is tangent to the ray $\Gamma_{\alpha}(s, \cdot)$ or, equivalently, where $\alpha$ is tangent to $T_{\alpha}^{f}(s, \cdot)$. We say that $s \in V_{\alpha}$ is transversal if the zero of the function $\Theta_{\alpha}(\cdot) \bmod \pi$ at $s$ is transversal.

2. There are $\alpha \in C$ such that $V_{\alpha}$ is a finite set containing transversal elements only except for 0 and 1 . We denote the subset of all such $\alpha \in C$ by $C^{f}$. In this case we find an order preserving, finite numbering of $V_{\alpha}$, i.e.

$$
V_{\alpha}=\left\{v_{1}, \ldots, v_{n}\right\} \quad \text { with } 0=v_{1}<\ldots<v_{n}=1 .
$$

In order to unify the notation we define $v_{0}:=v_{1}, v_{n}:=1$.

3. If $V_{\alpha}$ is a finite set then the sets

$$
(\angle f \circ \alpha \bmod 2 \pi)^{-1}(\varphi)
$$

are also finite for every angle $\varphi \in[0,2 \pi[$ (i.e. $f \circ \alpha$ has only finitely many intersections with every straight ray emanating from 0$)$. We can even find an angle $\omega_{\alpha} \in\left[0,2 \pi\left[\right.\right.$ such that $W_{\alpha} \cap V_{\alpha}=\emptyset$ with

$$
W_{\alpha}:=(\angle f \circ \alpha \bmod 2 \pi)^{-1}\left(\omega_{\alpha}\right) .
$$

Proof. 2. Lemma 1 shows that $C \neq \emptyset$. It is easy to see that arbitrarily close to every $\alpha_{0} \in C$ we find an $\alpha \in C$ such that the function $\Theta_{\alpha} \bmod \pi$ has only a finite number of transversal zeros. 
3. Because of statement 2 we know that $[0,1]=\left[v_{1}, v_{2}\right] \cup \ldots \cup\left[v_{n-1}, v_{n}\right]$. Lemma 2 shows that $\angle f \circ \alpha$ is strictly monotone on every $\left[v_{i}, v_{i+1}\right]$. Therefore $\left(\left|\angle f \circ \alpha\left(v_{i+1}\right)-\angle f \circ \alpha\left(v_{i}\right)\right| / 2 \pi\right)+1$ yields an upper bound on $(\angle f \circ \alpha$ $\bmod 2 \pi)^{-1}(\varphi) \cap\left[v_{i}, v_{i+1}\right]$ implying that $(\angle f \circ \alpha \bmod 2 \pi)^{-1}(\varphi)$ is finite itself. Since $\sharp V_{\alpha}$ is finite, so is $\sharp \angle f \circ \alpha\left(V_{\alpha}\right) \bmod 2 \pi$. Therefore, $\left[0,2 \pi\left[\backslash \angle f \circ \alpha\left(V_{\alpha}\right)\right.\right.$ $\bmod 2 \pi \neq \emptyset$, and $\omega_{\alpha}$ may be chosen to be any element of this set.

LEMMA 3. Let $s_{1}<s_{2}$ be two successive intersections of $\alpha$ with the image of a ray $\Gamma_{\alpha}^{f}(\bar{s}, \cdot)$. Then:

- if $s_{2}<1$ then $\left[s_{1}, s_{2}\left[\right.\right.$ contains an element of $V_{\alpha} \cup W_{\alpha}$,

- if $s_{2}=1$ then $\left[s_{1}, s_{2}\right]$ contains such an element.

Proof. If $s_{1}=0$ or $s_{2}=1$ we are done since $0,1 \in V_{\alpha}$. So assume that $s_{1}>0$ and $s_{2}<1$. Since $\alpha\left(s_{1}\right), \alpha\left(s_{2}\right) \in \operatorname{im} \Gamma_{\alpha}^{f}\left(\bar{s}_{1}, \cdot\right)$ we know that $f \circ \alpha\left(s_{1}\right), f \circ \alpha\left(s_{2}\right) \in \operatorname{im} \Gamma_{\alpha}\left(\bar{s}_{1}, \cdot\right)$. This implies that

$$
\angle f \circ \alpha\left(s_{1}\right)=\angle f \circ \alpha\left(s_{2}\right) \bmod 2 \pi
$$

(see Definition 2.4).

Assuming that there is no $s \in\left[s_{1}, s_{2}\left[\right.\right.$ with $s \in V_{\alpha}$, Lemma 2 shows that $\angle f \circ \alpha$ is strictly increasing (decreasing, resp.) in $\left[s_{1}, s_{2}\right]$. Therefore

$$
\angle f \circ \alpha\left(s_{1}\right) \neq \angle f \circ \alpha\left(s_{2}\right)
$$

and we deduce from (1) that

$$
\angle f \circ \alpha\left(s_{2}\right)-\angle f \circ \alpha\left(s_{1}\right)=2 \pi k
$$

with a $k \in \mathbb{Z} \backslash\{0\}$. Thus the continuity of $\angle f \circ \alpha$ implies that there must be an $s \in\left[s_{1}, s_{2}\left[\right.\right.$ such that $\angle f \circ \alpha(s)=\omega_{\alpha} \bmod 2 \pi$, i.e. $s \in W_{\alpha}$.

Definition\&Lemma 4. For all $s_{1}, s_{2} \in[0,1], s_{1}<s_{2}$, we define $n_{\alpha}\left(s_{1}, s_{2}\right) \in \mathbb{N}$ by

$$
n_{\alpha}\left(s_{1}, s_{2}\right):= \begin{cases}\sharp\left(\left[s_{1}, s_{2}\left[\cap\left(V_{\alpha} \cup W_{\alpha}\right)\right)\right.\right. & \text { if } s_{2}<1, \\ \sharp\left(\left[s_{1}, s_{2}\right] \cap\left(V_{\alpha} \cup W_{\alpha}\right)\right) & \text { if } s_{2}=1 .\end{cases}
$$

Then, for every $s \in] 0,1\left[\right.$ and every $\alpha \in C^{f}$,

$$
\sharp \alpha^{-1}\left(\operatorname{im} \Gamma_{\alpha}^{f}(s, \cdot)\right)-1 \leq n_{\alpha}\left(s_{\alpha}, s^{\alpha}\right),
$$

where

$$
s_{\alpha}:=\min \alpha^{-1}\left(\operatorname{im} \Gamma_{\alpha}^{f}(s, \cdot)\right), \quad s^{\alpha}:=\max \alpha^{-1}\left(\operatorname{im} \Gamma_{\alpha}^{f}(s, \cdot)\right)
$$

(i.e. the total number of intersections of $\alpha$ with the image of $\Gamma_{\alpha}^{f}(s, \cdot)$ minus 1 is at most the number of elements of $V_{\alpha} \cup W_{\alpha}$ which are between the first and the last intersection). Furthermore, we define $t_{\alpha}(s), t^{\alpha}(s) \in \Omega_{\alpha}(s)$ to be the unique elements such that

$$
\Gamma_{\alpha}^{f}\left(s, t_{\alpha}(s)\right)=\alpha\left(s_{\alpha}\right) \text { and } \quad \Gamma_{\alpha}^{f}\left(s, t^{\alpha}(s)\right)=\alpha\left(s^{\alpha}\right) .
$$


Proof. Since $\alpha \in C^{f}$ we know from Definition\&Lemma 3.2,3 that $\sharp \alpha^{-1}\left(\operatorname{im} \Gamma_{\alpha}^{f}(s, \cdot)\right)$ is also finite (see also Definition 2.4). Moreover, $s \in$ $\alpha^{-1}\left(\operatorname{im} \Gamma_{\alpha}^{f}(s, \cdot)\right)$ by definition. Thus $s_{\alpha}, s^{\alpha}$ are defined. Now, the assertion is a direct consequence of Lemma 3 .

Lemma 4. 1. $t_{\alpha}(s)=t^{\alpha}(s) \Rightarrow s_{\alpha}=s^{\alpha}$.

2. $s_{\alpha}=0$ and $s^{\alpha}=1$ at the same time is impossible. that

3. There is a neighbourhood $U_{\alpha}(s)$ of $\Gamma_{\alpha}^{f}\left(\{s\} \times\left[t_{\alpha}(s), t^{\alpha}(s)\right]\right)$ in $\mathbb{R}^{2}$ such

$$
f \mid U_{\alpha}(s): U_{\alpha}(s) \rightarrow f\left(U_{\alpha}(s)\right)
$$

is a diffeomorphism and $f\left(U_{\alpha}(s)\right)$ is a neighbourhood of $\Gamma_{\alpha}(\{s\} \times$ $\left.\left[t_{\alpha}(s), t^{\alpha}(s)\right]\right)$.

Proof. 1. $t_{\alpha}(s)=t^{\alpha}(s) \Rightarrow \alpha\left(s_{\alpha}\right)=\alpha\left(s^{\alpha}\right)$ (see the definition of $t_{\alpha}, t^{\alpha}$ in Definition\&Lemma 4$) \Rightarrow s_{\alpha}=s^{\alpha}$ since $\alpha$ is injective.

3. $\Gamma_{\alpha}(s, \cdot)$ is injective by definition, hence $f \mid \operatorname{im} \Gamma_{\alpha}^{f}(s, \cdot)$ is injective. If the assertion were false, we could construct two convergent (since $B:=\Gamma_{\alpha}^{f}(\{s\} \times$ $\left.\left[t_{\alpha}(s), t^{\alpha}(s)\right]\right) \subset \operatorname{im} \Gamma_{\alpha}^{f}(s, \cdot)$ is compact) sequences $x_{n} \rightarrow x \in B, y_{n} \rightarrow y \in B$ with $f\left(x_{n}\right)=f\left(y_{n}\right)$. However, this implies $f(x)=f(y)$, which is a contradiction since we already know that $f \mid B$ is injective.

2. From the definitions we conclude that $\alpha\left(s_{\alpha}\right), \alpha\left(s^{\alpha}\right) \in U_{\alpha}(s)$. If $s_{\alpha}=0$ and $s^{\alpha}=1$ then $\alpha\left(s_{\alpha}\right) \neq \alpha\left(s^{\alpha}\right)$ ( $\alpha$ is injective), hence assertion 3 implies that also $f \circ \alpha\left(s_{\alpha}\right) \neq f \circ \alpha\left(s^{\alpha}\right)$. However, $f \circ \alpha(0)=f \circ \alpha(1)$ by definition of $\alpha$-a contradiction.

Definition\&Lemma 5 (See Figure 1). Let us assume that $\alpha \in C^{f}$ and $\bar{s} \in] 0,1[$ are such that

$$
\sharp \alpha^{-1}\left(\operatorname{im} \Gamma_{\alpha}^{f}(\bar{s}, \cdot)\right) \geq 2 .
$$

Then we will construct a modified $\alpha$ (depending on $\bar{s}$ ), called $\alpha_{\bmod } \in C$ (associated with $\bar{s}$ ), such that

$$
n_{\alpha_{\text {mod }}}(0,1) \leq n_{\alpha}(0,1)-\left(\sharp \alpha^{-1}\left(\operatorname{im} \Gamma_{\alpha}^{f}(\bar{s}, \cdot)\right)-2\right) .
$$

First we define the curve $\beta_{0} \in C^{0}\left([0,1], \mathbb{R}^{2}\right)$ by

$$
\beta_{0}(s):= \begin{cases}f \circ \alpha(s) & \text { if } s \in\left[0, \bar{s}_{\alpha}\right] \cup\left[\bar{s}^{\alpha}, 1\right], \\ \Gamma_{\alpha}(\bar{s}, \tau(s)) & \text { if } s \in\left[\bar{s}_{\alpha}, \bar{s}^{\alpha}\right],\end{cases}
$$

with $\tau(s):=\left(s-\bar{s}_{\alpha}\right) t^{\alpha}(\bar{s}) /\left(\bar{s}^{\alpha}-\bar{s}_{\alpha}\right)+\left(s-\bar{s}^{\alpha}\right) t_{\alpha}(\bar{s}) /\left(\bar{s}_{\alpha}-\bar{s}^{\alpha}\right)$. Inequality (2) shows that $\bar{s}_{\alpha} \neq \bar{s}^{\alpha}$, hence $t_{\alpha}(\bar{s}) \neq t^{\alpha}(\bar{s})$. Therefore

1. $\beta_{0} \mid\left[\bar{s}_{\alpha}, \bar{s}^{\alpha}\right]$ is injective.

If we choose a neighbourhood $U_{\alpha}(\bar{s})$ of $\Gamma_{\alpha}^{f}\left(\{\bar{s}\} \times\left[t_{\alpha}(\bar{s}), t^{\alpha}(\bar{s})\right]\right)$ according to Lemma 4.3 we can also find a neighbourhood $\left[\bar{s}_{\alpha}-\varepsilon_{\alpha}, \bar{s}_{\alpha}+\varepsilon^{\alpha}\right]$ relative to $[0,1]$ such that: 
2. $\beta_{0}\left(\left[\bar{s}_{\alpha}-\varepsilon_{\alpha}, \bar{s}^{\alpha}+\varepsilon^{\alpha}\right]\right) \subset f\left(U_{\alpha}(\bar{s})\right)$, $\angle \beta_{0}\left(\left[\bar{s}_{\alpha}-\varepsilon_{\alpha}, \bar{s}^{\alpha}+\varepsilon^{\alpha}\right]\right) \subset\left[\angle \Gamma_{\alpha}(\bar{s}, 1)-\pi / 4, \angle \Gamma_{\alpha}(\bar{s}, 1)+\pi / 4\right] \bmod 2 \pi$.

3. $\beta_{0} \mid\left[\bar{s}_{\alpha}-\varepsilon_{\alpha}, \bar{s}^{\alpha}+\varepsilon^{\alpha}\right]$ is injective.

4. $\left[\bar{s}_{\alpha}-\varepsilon_{\alpha}, \bar{s}_{\alpha}\left[\cap\left(V_{\alpha} \cup W_{\alpha}\right)=\right] \bar{s}^{\alpha}, \bar{s}^{\alpha}+\varepsilon^{\alpha}\right] \cap\left(V_{\alpha} \cup W_{\alpha}\right)=\emptyset$.

At this stage we have to distinguish two cases:

Case 1: $\varepsilon_{\alpha}, \varepsilon^{\alpha}>0$ and

$$
\angle \beta_{0}\left(\bar{s}_{\alpha}-\varepsilon_{\alpha}\right)<\angle \beta_{0}\left(\bar{s}_{\alpha}\right)=\angle \beta_{0}\left(\bar{s}^{\alpha}\right)<\angle \beta_{0}\left(\bar{s}^{\alpha}+\varepsilon^{\alpha}\right)
$$

or vice versa, i.e. with " $<$ " replaced by " $>$ ".

Case 2: The condition for case 1 does not hold. This means that either $\bar{s}_{\alpha}=0$ or $\bar{s}^{\alpha}=1$ or in one of the two inequalities above we have " $>$ " and in the other we have " $<$ ".

In both cases we can find a $C^{1}$-curve $\beta$ arbitrarily close to $\beta_{0}$ such that:

5. $\beta(s)=\beta_{0}(s)=f \circ \alpha(s)$ if $s \in\left[0, \bar{s}_{\alpha}-\varepsilon_{\alpha}\right]$ or $s \in\left[\bar{s}^{\alpha}+\varepsilon^{\alpha}, 1\right]$.

6. $\beta\left(\left[\bar{s}_{\alpha}-\varepsilon_{\alpha}, \bar{s}^{\alpha}+\varepsilon^{\alpha}\right]\right) \subset f\left(U_{\alpha}(\bar{s})\right)$, $\angle \beta\left(\left[\bar{s}_{\alpha}-\varepsilon_{\alpha}, \bar{s}^{\alpha}+\varepsilon^{\alpha}\right]\right) \subset\left[\angle \Gamma_{\alpha}(\bar{s}, 1)-\pi / 4, \angle \Gamma_{\alpha}(\bar{s}, 1)+\pi / 4\right] \bmod 2 \pi$

(i.e. the modified part is contained in a half-cone).

7. $\dot{\beta}(s) \neq 0$ for all $s \in[0,1]$.

8. $\beta \mid\left[\bar{s}_{\alpha}-\varepsilon_{\alpha}, \bar{s}^{\alpha}+\varepsilon^{\alpha}\right]$ is injective.

9. In case 1 (case 2 , resp.) the function

$$
(\angle \dot{\beta}(\cdot)-\angle \beta(\cdot)) \bmod \pi
$$

has no zero (exactly one zero, resp.) in $\left[\bar{s}_{\alpha}-\varepsilon_{\alpha}, \bar{s}^{\alpha}+\varepsilon^{\alpha}\right]$. In case 2 this zero is transversal. (Remember that (3) being zero is the condition for $\beta$ being tangent at $s$ to a straight ray emanating from 0 .)

10. There is at most one $s \in\left[\bar{s}_{\alpha}-\varepsilon_{\alpha}, \bar{s}^{\alpha}+\varepsilon^{\alpha}\right]$ such that

$$
\angle \beta(s) \bmod 2 \pi=\omega_{\alpha}
$$

in case 1 and there is no such $s$ in case 2 .

Finally, we define $\alpha_{\bmod } \in C^{1}\left([0,1], \mathbb{R}^{2}\right)$ by

$$
\alpha \text { mod }(s):= \begin{cases}\left(f \mid U_{\alpha}(\bar{s})\right)^{-1} \circ \beta(s) & \text { if } s \in\left[\bar{s}_{\alpha}-\varepsilon_{\alpha}, \bar{s}^{\alpha}+\varepsilon^{\alpha}\right], \\ \alpha(s) & \text { if } s \in\left[0, \bar{s}_{\alpha}-\varepsilon_{\alpha}\right] \text { or } s \in\left[\bar{s}^{\alpha}+\varepsilon^{\alpha}, 1\right] .\end{cases}
$$

Then:

11. $\alpha_{\bmod } \in C$.

12. $n_{\alpha \text { mod }}(0,1) \leq n_{\alpha}(0,1)-\left(\sharp \alpha^{-1}\left(\operatorname{im} \Gamma_{\alpha}^{f}(\bar{s}, \cdot)-2\right)\right.$.

13. $V_{\alpha \bmod }$ has transversal elements only, i.e. $\alpha_{\bmod } \in C^{f}$.

14. $\angle f \circ \alpha \bmod \left(\left[\bar{s}_{\alpha}-\varepsilon_{\alpha}, \bar{s}^{\alpha}+\varepsilon^{\alpha}\right]\right) \subset\left[\angle \Gamma_{\alpha}(\bar{s}, 1)-\pi / 2, \angle \Gamma_{\alpha}(\bar{s}, 1)+\pi / 2\right] \bmod$ $2 \pi$ (i.e. the $f$-image of the modified part of $\alpha$ mod is contained in a half-cone).

Pro of. Assertion 1 is already proven above. 
If the neighbourhood $\left[\bar{s}_{\alpha}-\varepsilon_{\alpha}, \bar{s}^{\alpha}+\varepsilon^{\alpha}\right]$ is chosen to be small enough then assertion 2 is true by continuity, assertion 3 follows from assertion 1 and from the fact that $\beta_{0}$ is locally injective and assertion 4 is valid since $V_{\alpha}$ and $W_{\alpha}$ are finite sets.

Now using $2-3$, it is easy to see that there are $C^{1}$-curves $\beta$ arbitrarily close to $\beta_{0}$ which satisfy $5-10$. The precise construction is straightforward and is left to the reader since it would cause unproportionally much notation here.

11. Obviously, $\alpha_{\text {mod }}$ is a $C^{1}$-map and the properties (i) and (ii) required of $\alpha$ mod to be contained in $C$ are satisfied (see items 7 and 5). Moreover, since $\beta$ can be chosen to be arbitrarily close to $\beta_{0}$ property (iv) is also valid. It remains to prove that $\alpha$ mod is injective (property (iii)): In view of assertion 8 the definition of $\alpha$ mod shows that we only have to prove that the unchanged parts $\alpha$ mod $\mid\left[0, \bar{s}_{\alpha}-\varepsilon\right]$ and $\alpha$ mod $\mid\left[\bar{s}^{\alpha}+\varepsilon^{\alpha}, 1\right]$, resp. cannot intersect the modified part $\alpha$ mod $\mid\left[\bar{s}_{\alpha}-\varepsilon_{\alpha}, \bar{s}^{\alpha}+\varepsilon^{\alpha}\right]$. However, if this happened, then $\bar{s}_{\alpha}$ would not be the first intersection of $\alpha$ with $\operatorname{im} \Gamma_{\alpha}^{f}(\bar{s}, \cdot)$ or $\bar{s}^{\alpha}$ would not be the last, resp. (Use assertion 5 and again the fact that $\beta$ is arbitrarily close to $\beta_{0}$.)

12. Using 9-10 we conclude from Definition\&Lemma 3 and 4 that

$$
n_{\alpha \text { mod }}\left(\bar{s}_{\alpha}-\varepsilon_{\alpha}, \bar{s}^{\alpha}+\varepsilon^{\alpha}\right) \leq 1 .
$$

At the same time using Definition\&Lemma 4 we conclude from our hypothesis that

$$
n_{\alpha}\left(\bar{s}_{\alpha}, \bar{s}^{\alpha}\right) \geq \sharp \alpha^{-1}\left(\operatorname{im} \Gamma_{\alpha}^{f}(\bar{s}, \cdot)\right)-1 .
$$

Therefore, we may calculate

$$
\begin{aligned}
n_{\alpha_{\text {mod }}}(0,1)= & n_{\alpha_{\text {mod }}}\left(0, \bar{s}_{\alpha}-\varepsilon_{\alpha}\right)+n_{\alpha_{\text {mod }}}\left(\bar{s}_{\alpha}-\varepsilon_{\alpha}, \bar{s}^{\alpha}+\varepsilon^{\alpha}\right) \\
& +n_{\alpha_{\text {mod }}}\left(\bar{s}^{\alpha}+\varepsilon^{\alpha}, 1\right) \\
\leq & n_{\alpha_{\text {mod }}}\left(0, \bar{s}_{\alpha}-\varepsilon_{\alpha}\right)+1+n_{\alpha_{\text {mod }}}\left(\bar{s}^{\alpha}+\varepsilon_{\alpha}, 1\right)
\end{aligned}
$$

and

$$
\begin{aligned}
n_{\alpha}(0,1) & =n_{\alpha}\left(0, \bar{s}_{\alpha}\right)+n_{\alpha}\left(\bar{s}_{\alpha}, \bar{s}^{\alpha}\right)+n_{\alpha}\left(\bar{s}^{\alpha}, 1\right) \\
& \geq n_{\alpha}\left(0, \bar{s}_{\alpha}-\varepsilon_{\alpha}\right)+\sharp \alpha^{-1}\left(\operatorname{im} \Gamma_{\alpha}^{f}(\bar{s}, \cdot)\right)-1+n_{\alpha}\left(\bar{s}^{\alpha}+\varepsilon^{\alpha}, 1\right) .
\end{aligned}
$$

From the definition of $\alpha$ mod, assertion 12 follows.

Finally assertion 13 follows from 9, and 14 from 6 .

Definition\&Lemma 6 (See Figure 1 ). For every $\bar{s} \in] 0,1[$ and every curve $\alpha \in C^{f}$ we define

$$
\mu_{\alpha}(s):= \begin{cases}2 & \text { if } s \in] 0,1\left[\cap V_{\alpha}\right. \\ 1 & \text { otherwise }\end{cases}
$$

for all $s \in S_{\alpha}(\bar{s}):=\alpha^{-1}\left(\operatorname{im} \Gamma_{\alpha}^{f}(\bar{s}, \cdot)\right)$. There exists a curve $\alpha_{D} \in C^{f}$ such 
that

$$
\sum_{s \in S_{\alpha_{D}}(\bar{s})} \mu_{\alpha_{D}}(s) \leq 2
$$

for all $\bar{s} \in] 0,1\left[\right.$. (This means that the number of intersections of $\alpha_{D}$ with $\operatorname{im} \Gamma_{\alpha_{D}}^{f}(\bar{s}, \cdot)$ is at most two. Interior intersections which are tangential at the same time are counted twice.)

Proof. We construct the desired $\alpha_{D}$ by a finite iteration of the modification process of Definition\&Lemma 5:

We start with an arbitrary $\alpha \in C^{f}$. (By Definition\&Lemma 3.2 this set is non-empty.) If $\sum_{s \in S_{\alpha}(\bar{s})} \mu_{\alpha}(s)>2$ for some $\left.\bar{s} \in\right] 0,1\left[\right.$ and if $\sharp S_{\alpha}(\bar{s}) \leq 2$ there must be at least one $s \in S_{\alpha}(\bar{s})$ with $\mu(s)=2$, i.e. $\left.s \in\right] 0,1\left[\cap V_{\alpha}\right.$. In other words, $\alpha$ intersects $\operatorname{im} \Gamma_{\alpha}^{f}(\bar{s}, \cdot)$ at $s$ tangentially and the function $\Theta_{\alpha}(\cdot) \bmod \pi$ has a transversal zero at $s$ (since $\left.s \in\right] 0,1\left[\cap V_{\alpha}\right.$ and $\alpha \in C^{f}$ ). This implies that the tangential intersection of $\alpha$ with $\operatorname{im} \Gamma_{\alpha}^{f}(\bar{s}, \cdot)$ at $s$ is non-transversal. Therefore we can slightly modify $\alpha$ in a neighbourhood of $s$ such that this intersection bifurcates into two intersections and such that $V_{\alpha}$ and $W_{\alpha}\left(\alpha \in C^{f} \Rightarrow s \notin W_{\alpha}\right.$, see Definition\&Lemma 3.3) remain unchanged.

Thus we may assume without loss of generality that $\sharp S_{\alpha}(\bar{s})>2$. Now an application of Definition\&Lemma 5.12 yields an $\alpha_{\bmod } \in C^{f}$ such that $n_{\alpha_{\text {mod }}}(0,1) \leq n_{\alpha}(0,1)-1$.

Repeating this process if necessary we will finally arrive (since $n_{\alpha}(0,1)$ cannot become negative) at an $\alpha_{D} \in C^{f}$ such that the assertion is valid.

Now we define a map (Definition 3) which relates the remaining two intersections of $\alpha_{D}$ with a lifted ray if they exist. Lemma 5 summarizes the most important properties of this map.

Definition 3. Set $A:=\{s \in] 0,1\left[\mid \sharp \alpha_{D}^{-1}\left(\operatorname{im} \Gamma_{\alpha_{D}}^{f}(s, \cdot)\right) \geq 2\right\}$. Since always $s \in \alpha_{D}^{-1}\left(\operatorname{im} \Gamma_{\alpha_{D}}^{f}(s, \cdot)\right)$ and by Definition\&Lemma 6 we may define the following maps:

$$
a: A \rightarrow[0,1], \quad a(s):=\alpha_{D}^{-1}\left(\operatorname{im} \Gamma_{\alpha_{D}}^{f}(s, \cdot)\right) \backslash\{s\}
$$

(where we have identified one-element sets with their element) and $b: A \rightarrow$ $\left[0, \infty\left[\right.\right.$ with $b(s)$ being the unique (since $\Gamma_{\alpha_{D}}^{f}(s, \cdot)$ is injective) element of $\Omega_{\alpha_{D}}(s)$ such that

$$
\alpha_{D}(a(s))=\Gamma_{\alpha_{D}}^{f}(s, b(s)) .
$$

Lemma 5. 1. $\left.a(A) \cap V_{\alpha_{D}} \cap\right] 0,1\left[=\emptyset, A \cap V_{\alpha_{D}}=\emptyset\right.$.

2. $A$ is open and $a, b$ are continuous. In particular, $a^{-1}(0), a^{-1}(1)$ are also open.

3. If $a\left(s_{1}\right)=a\left(s_{2}\right) \notin\{0,1\}$ then $s_{1}=s_{2}$. Moreover, $a$ is monotone on every interval $I \subset A$ and is strictly monotone if $0,1 \notin a(I)$. In addition, $b(s) \neq 1$ everywhere. 
4. $\angle f \circ \alpha_{D}$ and $\angle f \circ \alpha_{D} \circ a$ are monotone on every interval $I \subset A$.

5. For all $i=1, \ldots, n-1$ there is an $\varepsilon>0$ such that $] v_{i}, v_{i}+\varepsilon[\subset A$ (]$v_{i+1}-\varepsilon, v_{i+1}[\subset A$, resp. $)$. Moreover,

$$
\begin{aligned}
a(s) \leq v_{i} \quad \text { and } \quad \text { either } b(s) & <1 \text { if } \Theta_{\alpha_{D}}\left(v_{i}\right)=0 \bmod 2 \pi, \\
\text { or } b(s) & >1 \text { if } \Theta_{\alpha_{D}}\left(v_{i}\right)=\pi \bmod 2 \pi \\
\left(\text { or } a(s) \geq v_{i+1} \quad \text { and } \quad \text { either } b(s)\right. & <1 \text { if } \Theta_{\alpha_{D}}\left(v_{i+1}\right)=\pi \bmod 2 \pi, \\
\text { or } b(s) & >1 \text { if } \Theta_{\alpha_{D}}\left(v_{i+1}\right)=0 \bmod 2 \pi, \text { resp.) }
\end{aligned}
$$

for all $s \in] v_{i}, v_{i}+\varepsilon\left[(\in] v_{i+1}-\varepsilon, v_{i+1}[\right.$, resp. $)$. In addition,

$$
\lim _{s \rightarrow v_{i}} a(s)=v_{i} \quad\left(\lim _{s \rightarrow v_{i+1}} a(s)=v_{i+1}, \text { resp. }\right) .
$$

6. Let $I \subset A$ be an interval such that $v_{i} \in \bar{I}$ for some $i=1, \ldots, n$. Then, for all $s \in I$,

$$
\angle f \circ \alpha_{D} \circ a(s) \leq(\geq \text {,resp. }) \angle f \circ \alpha_{D}(s)
$$

if $v_{i}=\inf I\left(v_{i}=\sup I\right.$, resp. $)$ and $\angle f \circ \alpha_{D}$ is increasing on $I$, or if $v_{i}=\sup I$ $\left(v_{i}=\inf I\right.$, resp. $)$ and $\angle f \circ \alpha_{D}$ is decreasing on $I$.

Proof. 1. If we assume that $\left.a(s) \in V_{\alpha_{D}} \cap\right] 0,1\left[\right.$ then $\mu_{\alpha_{D}}(s)=2$ and Definition\&Lemma 6 shows that $a(s)$ must be the only element of $\alpha_{D}^{-1}\left(\operatorname{im} \Gamma_{\alpha_{D}}^{f}(s, \cdot)\right)$. However, $s \in \alpha_{D}^{-1}\left(\operatorname{im} \Gamma_{\alpha_{D}}^{f}(s, \cdot)\right)$ by definition-a contradiction.

If we assume that $s \in V_{\alpha_{D}}$ then $\mu_{\alpha_{D}}(s)=2$ or $s \in\{0,1\}$. Therefore, Definition\&Lemma 6 shows again that either $\alpha_{D}^{-1}\left(\operatorname{im} \Gamma_{\alpha_{D}}^{f}(s, \cdot)\right) \backslash\{s\}=\emptyset$ or $s \in\{0,1\}$. Both cases imply that $s \notin A$.

2. If $\alpha_{D}(a(s))=\Gamma_{\alpha_{D}}^{f}(s, b(s))$ then assertion 1 shows that either $a(s) \in$ $\{0,1\}$ or $a(s) \notin V_{\alpha_{D}}$. We recall that $\Omega_{\alpha_{D}}$ is open in $] 0,1[\times[0, \infty[$.

In the first case we take $a\left(s^{\prime}\right):=a(s) \in\{0,1\}$ for all $s \in[0,1]$. Then the implicit function theorem yields a continuous extension of $b$ to some neighbourhood of $s$ since $\left(\Gamma_{\alpha_{D}}^{f}(s, \cdot)\right)^{\cdot} \neq 0$.

In the second case we directly apply the implicit function theorem and obtain a continuous extension of $a$ and $b$ to some neighbourhood of $s$. This is due to the fact that $\dot{a}(s)$ and $\left(\Gamma_{\alpha_{D}}^{f}(s, \cdot)\right)^{\cdot}$ are not parallel if $a(s) \notin V_{\alpha_{D}}$.

3 . Since $a\left(s_{1}\right)=a\left(s_{2}\right) \notin\{0,1\}$ we conclude that

$$
\Gamma_{\alpha_{D}}^{f}\left(s_{1}, b\left(s_{1}\right)\right)=\alpha_{D}\left(a\left(s_{1}\right)\right)=\alpha_{D}\left(a\left(s_{2}\right)\right)=\Gamma_{\alpha_{D}}^{f}\left(s_{2}, b\left(s_{2}\right)\right)
$$

with $b\left(s_{1}\right), b\left(s_{2}\right) \neq 0$. Thus it is clear that $\operatorname{im} \Gamma_{\alpha_{D}}^{f}\left(s_{1}, \cdot\right)=\operatorname{im} \Gamma_{\alpha_{D}}^{f}\left(s_{2}, \cdot\right)$. Therefore, if $s_{1} \neq s_{2}$ then $\operatorname{im} \Gamma_{\alpha_{D}}^{f}\left(s_{1}, \cdot\right)$ would contain three different points, namely $s_{1}, s_{2}$ and $a\left(s_{1}\right)=a\left(s_{2}\right)$. This contradicts Definition\&Lemma 6 .

Now, monotonicity is obvious if we also use the connectedness of $I$ and the continuity of $a$.

If $b(s)=1$ then $\alpha_{D}(a(s))=\Gamma_{\alpha_{D}}^{f}(s, b(s))=\Gamma_{\alpha_{D}}^{f}(s, 1)=\alpha_{D}(s)$. Since $\alpha_{D}$ is injective we conclude that $a(s)=s$, a contradiction. 
4. Assertion 1 implies that neither $I$ nor $a(I)$ contain an element of $V_{\alpha_{D}}$ in their interior (in $\mathbb{R}$ ). Therefore Lemma 2 shows that $\angle f \circ \alpha_{D}$ is monotone on $I$ and on $a(I)$. Since $a$ is also monotone (see assertion 3 ) we are done.

5. Since the assertion is local and $f$ is a local diffeomorphism we may consider the $f$-images of $\alpha_{D}$ and $\Gamma_{\alpha_{D}}^{f}$ as well. Thus if $v_{i+1}=0$ it is easy to see that the assertion is true with $a(s)=0$ and $b(s)=0$ since $f \circ \alpha_{D}(0)=$ $\Gamma_{\alpha_{D}}(s, 0)$.

If $v_{i+1} \neq 0$ then it is not difficult to see that the situation in a neighbourhood of $f \circ \alpha_{D}\left(v_{i}\right)$ is homeomorphic to the following one on $] 0,1\left[^{2}\right.$ : Let

$$
\begin{aligned}
f \circ \alpha_{D}(s) & :=\left((s-1 / 2)^{2}, s-1 / 2\right), \\
\Gamma_{\alpha_{D}}(s, t) & :=\left((s-1 / 2)^{2},(s-1 / 2)+t-1\right)
\end{aligned}
$$

and $v_{i+1}=1 / 2\left(\right.$ case $\left.\Theta_{\alpha_{D}}\left(v_{i+1}\right)=0 \bmod 2 \pi\right)$. Then

$$
f \circ \alpha_{D}(a(s))=\Gamma_{\alpha_{D}}(s, b(s))
$$

with $a(s):=1-s, b(s):=2-2 s$. Now it is straightforward to verify the assertion. (We have only treated the assertion at $v_{i+1}$ with $\Theta_{\alpha_{D}}\left(v_{i+1}\right)=$ $0 \bmod 2 \pi$. However, the other cases are completely analogous.)

6. $I$ may always be partitioned into $I=I_{0} \cup I^{\prime} \cup I_{1}$ with

$$
I_{0}:=a^{-1}(0) \cap I, \quad I^{\prime}:=a^{-1}(] 0,1[) \cap I, \quad I_{1}:=a^{-1}(1) \cap I .
$$

However, since $a$ is monotone on $I$ we know that $I_{0}, I^{\prime}, I_{1}$ are intervals with

$$
\sup I_{0}=\inf I^{\prime}, \quad \sup I^{\prime}=\inf I_{1}
$$

if $a$ is increasing, and

$$
\sup I_{1}=\inf I^{\prime}, \quad \sup I^{\prime}=\inf I_{0}
$$

if $a$ is decreasing on $I$. In addition, at least one of the intervals $I_{0}, I_{1}$ is always empty. Otherwise, using the intermediate value theorem we could find an $s \in I$ with $s=a(s)$, a contradiction.

Our definitions show that

$$
\angle f \circ \alpha_{D} \circ a(s)= \begin{cases}\angle f \circ \alpha_{D}(0) & \text { if } s \in I_{0}, \\ \angle f \circ \alpha_{D}(s) \bmod 2 \pi & \text { if } s \in I^{\prime}, \\ \angle f \circ \alpha_{D}(1) & \text { if } s \in I_{1} .\end{cases}
$$

We treat the case when $v_{i}=\inf I$ and $\angle f \circ \alpha_{D}$ is increasing on $I$ :

Assertion 5 (i.e. $a(s) \leq v_{i}$ and $\lim _{s \rightarrow v_{i}} a(s)=v_{i}$ ) shows that $a$ is decreasing. Therefore, we know that either $I=I_{1} \cup I^{\prime}$ or $I=I^{\prime} \cup I_{0}$ with (5) being satisfied.

If $I=I_{1} \cup I^{\prime}$ (with $I_{1}$ assumed to be non-empty) then

$$
\inf I=v_{i}=\lim _{s \rightarrow v_{i}} a(s)=1 \text {. }
$$


At the same time $I \subset] 0,1[$ by definition. Therefore, $I$ must be empty - a contradiction.

If $I=I^{\prime} \cup I_{0}$ then assertion 5 shows that

$$
\lim _{s \rightarrow v_{i}} \angle f \circ \alpha_{D} \circ a(s)=\lim _{s \rightarrow v_{i}} \angle f \circ \alpha_{D}(s) .
$$

Therefore, (6) shows that

$$
\angle f \circ \alpha_{D} \circ a(s)=\angle f \circ \alpha_{D}(s)
$$

for all $s \in I^{\prime}$ by continuation.

If $s \in I_{0}$ we calculate (using the fact that $f \circ \alpha_{D}$ is increasing):

$$
\begin{aligned}
\angle f \circ \alpha_{D}(s) & \geq \angle f \circ \alpha_{D}\left(\inf I_{0}\right)=\angle f \circ \alpha_{D} \circ a\left(\inf I_{0}\right) \\
& =\angle f \circ \alpha_{D}(0)=\angle f \circ \alpha_{D} \circ a(s),
\end{aligned}
$$

where the first equality follows from (7) by continuity. We also used (6).

The other three cases are completely analogous.

The following lemma shows that without loss of generality we may assume that the remaining "oscillations" (i.e. the variation of $\angle f \circ \alpha_{D}$ ) are not too large in some sense.

LEMMA 6. Without loss of generality we may assume that for all intervals $I \subset A$ such that $v_{i} \in \bar{I}$ (closure of $I$ in $\mathbb{R}$ ) (for some $i=1, \ldots, n$ ) and for all $\bar{s} \in I$,

$$
\left|\angle f \circ \alpha_{D}(\bar{s})-\angle f \circ \alpha_{D}\left(v_{i}\right)\right| \leq \pi .
$$

Proof. If the assertion is not true for a $v_{i}$, an $I$ and an $\bar{s}$ we use Definition\&Lemma 5 in order to replace $\alpha_{D}$ by its modification $\left(\alpha_{D}\right)$ mod associated with $\bar{s}$. (This is possible since $\bar{s} \in I$ implies $\sharp \alpha_{D}^{-1}\left(\operatorname{im} \Gamma_{\alpha_{D}}^{f}(\bar{s}, \cdot)\right)=2$.) Clearly, all properties of $\alpha_{D}$ are conserved.

We show that this reduces

$$
\operatorname{Var}\left(\alpha_{D}\right):=\sum_{k=1}^{n-1}\left|\angle f \circ \alpha_{D}\left(v_{k+1}\right)-\angle f \circ \alpha_{D}\left(v_{k}\right)\right|
$$

by $\pi / 2$ at least. Since $v_{i} \in \bar{I}$ we conclude from Lemma 5 that $a(\bar{s}) \in\left[v_{i-1}, v_{i}\right]$ (or $a(\bar{s}) \in\left[v_{i}, v_{i+1}\right]$, resp.).

Without loss of generality we will consider only the first case further on. Moreover, $\sharp \alpha_{D}^{-1}\left(\operatorname{im} \Gamma_{\alpha_{D}}^{f}(\bar{s}, \cdot)\right)=2$ shows that $\bar{s}_{\alpha}=\bar{s}$ and $\bar{s}^{\alpha}=a(\bar{s})$ or vice versa. Since $\left(\alpha_{D}\right)$ mod equals $\alpha_{D}$ except for all $\left.s \in\right] \bar{s}_{\alpha}-\varepsilon_{\alpha}, \bar{s}^{\alpha}+\varepsilon^{\alpha}[$ (with $\bar{s}_{\alpha}=\bar{s}$ and $\bar{s}^{\alpha}=a(\bar{s})$ or vice versa) only the (at most two) summands of $\operatorname{Var}\left(\alpha_{D}\right)$ which contain $v_{i}$ are affected by the modification.

Thus we calculate (using the abbreviation $\check{s}:=\angle f \circ \alpha_{D}(s)$ and recalling that we have defined $\left.v_{0}:=v_{1}=0, v_{n+1}:=v_{n}=1\right)$ :

$$
\left|\angle f \circ \alpha_{D}\left(v_{i}\right)-\angle f \circ \alpha_{D}\left(v_{i-1}\right)\right|+\left|\angle f \circ \alpha_{D}\left(v_{i+1}\right)-\angle f \circ \alpha_{D}\left(v_{i}\right)\right|
$$




$$
\begin{aligned}
& \stackrel{(1)}{=}\left|\check{v}_{i}-\check{a}(\bar{s})\right|+\left|\check{a}(\bar{s})-\check{v}_{i-1}\right|+\left|\check{v}_{i+1}-\check{\bar{s}}\right|+\left|\check{\bar{s}}-\check{v}_{i}\right| \\
& \stackrel{(2)}{>} 0+\left|\check{a}(\bar{s})-\check{v}_{i-1}\right|+\left|\check{v}_{i+1}-\check{\bar{s}}\right|+\pi \\
& \stackrel{(3)}{\geq}\left|\check{a}(\bar{s})-\check{v}_{i-1}\right|+\left|\check{v}_{i+1}-\check{\bar{s}}\right|+2\left|\check{\bar{s}}-\check{v}_{i, \bmod }\right|+\pi / 2 \\
& \stackrel{(4)}{\geq}\left|\check{v}_{i, \bmod }-\check{a}(\bar{s})\right|+\left|\check{a}(\bar{s})-\check{v}_{i-1}\right|+\left|\check{v}_{i+1}-\check{\bar{s}}\right|+\left|\check{\bar{s}}-\check{v}_{i, \bmod }\right|+\pi / 2 \\
& \stackrel{(5)}{=}\left|\check{v}_{i, \bmod }-\check{v}_{i-1}\right|+\left|\check{v}_{i+1}-\check{v}_{i, \bmod }\right|+\pi / 2 .
\end{aligned}
$$

Here, $v_{i}$, mod denotes the single element of $V_{\alpha_{D}}$ which is affected by the modification. Equation (1) holds since $\check{a}(\bar{s}) \in\left[v_{i-1}, v_{i}\right]$ and $\bar{s} \in\left[v_{i}, v_{i+1}\right]$ and since $\angle f \circ \alpha_{D}$ and $\angle f \circ \alpha_{D} \circ a$ are monotone (see Lemma 5).

Inequality (2) follows from our assumption that the assertion is not true.

Inequality (3) uses Definition\&Lemma 5.14, and inequality (4) is a consequence of Lemma 5.6. Equation (5) is similar to equation (1).

Thus, our calculation shows that each time we apply this modification, we reduce $\operatorname{Var}\left(\alpha_{D}\right)$ by $\pi / 2$ at least. Since $\operatorname{Var}\left(\alpha_{D}\right)$ cannot become negative, we will finally arrive at a curve which satisfies the assertion.

Now we aim at constructing an unbounded, injective curve $\gamma$ such that the tangent angle of its image $f \circ \gamma$ rotates by an amount of $3 \pi+\varepsilon(\varepsilon>0)$ at least if we follow the curve from a point close to the first "end" of $\gamma$ to a point close to the other "end" (see Definition\&Lemma 8). To this end we isolate an appropriate part of $\alpha_{D}$, namely $\alpha_{D} \mid\left[a_{1}, a_{2}\right]$ (Definition 4, Lemma 8) and hang on the lifted rays which pass through its ends (Definition\&Lemma 7 and Lemma 9). Lemma 7 ensures that the above mentioned rotation is $3 \pi$ at least. Definition\&Lemma 8 adds the $\varepsilon$-summand. This will be done by a slight rotation of one of the added rays.

Lemma 7 (See Figure 1). There are successive $v_{k}, v_{k+1} \in V_{\alpha_{D}}$ with $k \in$ $\{1, \ldots, n-1\}$ such that $\Theta_{\alpha_{D}}\left(v_{k}\right) \bmod 2 \pi=0$ and either

$$
\Theta_{\alpha_{D}}\left(v_{k+1}\right)=\Theta_{\alpha_{D}}\left(v_{k}\right)+\pi, \quad \angle f \circ \alpha_{D}\left(v_{k}\right)<\angle f \circ \alpha_{D}\left(v_{k+1}\right)
$$

or

$$
\Theta_{\alpha_{D}}\left(v_{k+1}\right)=\Theta_{\alpha_{D}}\left(v_{k}\right)-\pi, \quad \angle f \circ \alpha_{D}\left(v_{k}\right)>\angle f \circ \alpha_{D}\left(v_{k+1}\right) .
$$

Throughout the rest of this work we assume without loss of generality that the first alternative holds. This can always be achieved by an orientation reversing reparametrization if necessary. Moreover, $k$ will be fixed from now on according to this lemma.

Proof. From Definition\&Lemma 2.2 we know that $\Theta_{\alpha_{D}}(0) \bmod 2 \pi=$ 0 . If $v_{k}, v_{k+1} \in V_{\alpha_{D}}$ are successive the continuity of $\Theta_{\alpha_{D}}$ implies that $\left|\Theta_{\alpha_{D}}\left(v_{k+1}\right)-\Theta_{\alpha_{D}}\left(v_{k}\right)\right|$ must either equal $\pi$ or equal 0 . 
If the latter were true for every successive pair $v_{i}, v_{i+1} \in V_{\alpha_{D}}$ we could prove iteratively that $\Theta_{\alpha_{D}}(1)=\Theta_{\alpha_{D}}(0)$ (remember that $0,1 \in V_{\alpha_{D}}$ ), which contradicts Definition\&Lemma 2.2. Therefore, there is a first successive pair $v_{i}, v_{i+1}$ such that $\left|\Theta_{\alpha_{D}}\left(v_{i+1}\right)-\Theta_{\alpha_{D}}\left(v_{i}\right)\right|=\pi$. For this pair, the first two parts of the assertion (i.e. those concerning $\Theta_{\alpha_{D}}$ ) are obviously satisfied. The last part of the assertion is now a direct consequence of Lemma 2.

DEFINITION 4.

$$
\left.A_{1}:=a^{-1}\left(\left[v_{k-1}, v_{k}\right]\right) \cap\right] v_{k}, v_{k+1}\left[, \quad A_{2}:=a^{-1}\left(\left[v_{k+1}, v_{k+2}\right]\right) \cap\right] v_{k}, v_{k+1}[.
$$

(Notice that $\left[v_{k-1}, v_{k}\right]=\{0\}$ if $k=1$ as well as $\left[v_{k+1}, v_{k+2}\right]=\{1\}$ if $k+1=n$ since we have set $v_{0}:=v_{1}, v_{n+1}:=v_{n}$.)

Lemma 8. 1. $A_{1} \cap A_{2}=\emptyset$.

2. $A_{1}, A_{2} \neq \emptyset$. In particular, there are $\varepsilon_{1}, \varepsilon_{2}>0$ such that $] v_{k}, v_{k}+\varepsilon_{1}[\subset$ $A_{1}$ and $] v_{k+1}-\varepsilon_{2}, v_{k+2}\left[\subset A_{2}\right.$.

3. $A_{1}, A_{2}$ are open in $] v_{k}, v_{k+1}[$.

4. There is an $\bar{r} \in] v_{k}, v_{k+1}\left[\right.$ such that $\bar{r} \notin A_{1} \cup A_{2}$.

Proof. 1. This is clear since $\left[v_{k-1}, v_{k}\right] \cap\left[v_{k+1}, v_{k+2}\right]=\emptyset$.

2. This is obvious from Lemma 5.5.

3. Lemma 5.1 shows that

$$
a^{-1}\left(\left[v_{k-1}, v_{k}\right]\right)= \begin{cases}a^{-1}(] v_{k-1}, v_{k}[) & \text { if } k>2, \\ a^{-1}\left(\left[0, v_{2}[)\right.\right. & \text { if } k=2, \\ a^{-1}(0) & \text { if } k=1 .\end{cases}
$$

Now Lemma 5.2 implies that $A_{1}$ is open. The proof for $A_{2}$ is analogous.

4. Since $] v_{k}, v_{k+1}[$ is connected, $1-3$ show that $] v_{k}, v_{k+1}\left[\backslash\left(A_{1} \cup A_{2}\right)\right.$ must be non-empty.

Definition\&Lemma 7 (See Figure 1). Let $r_{1}$ be the smallest element of $] v_{k}, v_{k+1}\left[\backslash A_{1}\right.$ and $r_{2}$ be the greatest element of $] v_{k}, v_{k+1}\left[\backslash A_{2}\right.$. They exist since these sets are left (right, resp.) closed (see Lemma 8.2, 3) and since ]$v_{k}, v_{k+1} \backslash \backslash\left(A_{1} \cup A_{2}\right) \neq 0$ (see Lemma 8.4). Moreover,

$$
v_{k}<r_{1} \leq \bar{r} \leq r_{2}<v_{k+1} .
$$

Let $r_{1}^{n} \rightarrow r_{1}$ and $r_{2}^{n} \rightarrow r_{2}$ be sequences in $A_{1}, A_{2}$, resp. We define

$$
\begin{array}{ll}
a_{1}:=\lim _{n \rightarrow \infty} a\left(r_{1}^{n}\right) \in\left[v_{k-1}, v_{k}\right], & b_{1}:=\lim _{n \rightarrow \infty} b\left(r_{1}^{n}\right), \\
a_{2}:=\lim _{n \rightarrow \infty} a\left(r_{2}^{n}\right) \in\left[v_{k+1}, v_{k+2}\right], & b_{2}:=\lim _{n \rightarrow \infty} b\left(r_{2}^{n}\right) .
\end{array}
$$

(These limits exist for a suitable subsequence at least.) By continuity,

$$
\Gamma_{\alpha_{D}}\left(r_{1}, b_{1}\right)=f \circ \alpha_{D}\left(a_{1}\right), \quad \Gamma_{\alpha_{D}}\left(r_{2}, b_{2}\right)=f \circ \alpha_{D}\left(a_{2}\right) .
$$

Let $\Gamma_{\alpha_{D}}^{f, a_{1}}\left(r_{1}, \cdot\right)\left(\Gamma_{\alpha_{D}}^{f, a_{2}}\left(r_{2}, \cdot\right)\right.$, resp.) denote the lift of $\Gamma_{\alpha_{D}}\left(r_{1}, \cdot\right)\left(\Gamma_{\alpha_{D}}\left(r_{2}, \cdot\right)\right.$, resp.) with respect to $f$ such that $\Gamma_{\alpha_{D}}\left(r_{1}, b_{1}\right)\left(\Gamma_{\alpha_{D}}\left(r_{2}, b_{2}\right)\right.$, resp. $)$ is lifted to 
$\alpha_{D}\left(a_{1}\right)\left(\alpha_{D}\left(a_{2}\right)\right.$, resp.). Moreover, let $\left.\Omega_{\alpha_{D}}^{a_{1}}\left(r_{1}\right)=\right] c_{1}, d_{1}\left[\left(\Omega_{\alpha_{D}}^{a_{2}}\left(r_{2}\right)=\right] c_{2}, d_{2}[\right.$, resp.) denote the maximal interval of existence. (Of course $b_{1} \in \Omega_{\alpha_{D}}^{a_{1}}\left(r_{1}\right), b_{2}$ $\in \Omega_{\alpha_{D}}^{a_{2}}\left(r_{2}\right)$.) Then:

1. $\alpha_{D}\left(\left[a_{1}, a_{2}\right]\right) \cap \Gamma_{\alpha_{D}}^{f, a_{1}}\left(\left\{r_{1}\right\} \times\left[b_{1}, d_{1}[)=\alpha_{D}\left(a_{1}\right)\right.\right.$, $\alpha_{D}\left(\left[a_{1}, a_{2}\right]\right) \cap \Gamma_{\alpha_{D}}^{f, a_{2}}\left(\left\{r_{2}\right\} \times\left[b_{2}, d_{2}[)=\alpha_{D}\left(a_{2}\right)\right.\right.$.

2. $\Gamma_{\alpha_{D}}^{f, a_{1}}\left(\left\{r_{1}\right\} \times\left[b_{1}, d_{1}[) \cap \Gamma_{\alpha_{D}}^{f, a_{2}}\left(\left\{r_{2}\right\} \times\left[b_{2}, d_{2}[)=\emptyset\right.\right.\right.\right.$.

3. $b_{1} \leq 1, b_{2} \leq 1$.

Pro of. 1 . We assume the contrary, i.e. there are an $\left.s \in] a_{1}, a_{2}\right]$ and a $t \in$ $\left[b_{1}, \infty\left[\right.\right.$ such that $\alpha_{D}(s)=\Gamma_{\alpha_{D}}^{f, a_{1}}\left(r_{1}, t\right)$. Then this intersection is transversal:

Indeed, since already $\alpha_{D}\left(a_{1}\right)=\Gamma_{\alpha_{D}}^{f, a_{1}}\left(r_{1}, b_{1}\right)$ we conclude from Definition\&Lemma 6 that $s \notin] 0,1\left[\cap V_{\alpha_{D}}\right.$. Our assumption says that $s \neq 0$. Assume $s=1$. Then $f \circ \alpha_{D}(s)=0$, hence $t=0$ and therefore $b_{1}=0$. However, the latter implies that $a_{1}=0$ and we arrive at a contradiction to Lemma 4.2 (with $s_{\alpha}=a_{1}, s^{\alpha}=s$ ).

Thus we see that $s \in V_{\alpha_{D}}$ is impossible and therefore our intersection is transversal as asserted. Therefore, using the fact that $\Omega_{\alpha_{D}}$ (see Definition 2 ) is open, we conclude that this intersection continues to the lifts of nearby rays, i.e. to all lifted rays $\Gamma_{\alpha_{D}}^{f, a\left(r_{1}^{n}\right)}\left(r_{1}^{n}, \cdot\right)$ with $n$ large enough.

Thus we deduce that $\alpha_{D}$ intersects $\operatorname{im} \Gamma_{\alpha_{D}}^{f, a\left(r_{1}^{n}\right)}\left(r_{1}^{n}, \cdot\right)$ for all large $n$ not only at $r_{1}^{n}$ and at $a\left(r_{1}^{n}\right)$ but also at some $s_{n}$ with $s_{n} \rightarrow s$. We know that $r_{1}^{n} \rightarrow r_{1} \neq a_{1}, a\left(r_{1}^{n}\right) \rightarrow a_{1}, s_{n} \rightarrow s \neq a_{1}$. If we also assume that $s \neq r_{1}$ for the moment we deduce that $r_{1}^{n}, a\left(r_{1}^{n}\right), s_{n}$ are pairwise different for all large $n$. This yields a contradiction to Definition\&Lemma 6 .

Now we are left with proving that $s \neq r_{1}$. From our assumption $\alpha_{D}(s) \in$ $\operatorname{im} \Gamma_{\alpha_{D}}^{f, a_{1}}\left(r_{1}, \cdot\right)$ we conclude that $\operatorname{im} \Gamma_{\alpha_{D}}^{f, a_{1}}\left(r_{1}, \cdot\right)=\operatorname{im} \Gamma_{\alpha_{D}}^{f}(s, \cdot)$. Therefore, if $s=r_{1}$ it would follow that

$$
\alpha_{D}\left(a_{1}\right) \in \operatorname{im} \Gamma_{\alpha_{D}}^{f, a_{1}}\left(r_{1}, \cdot\right)=\operatorname{im} \Gamma_{\alpha_{D}}^{f}\left(r_{1}, \cdot\right),
$$

i.e. $r_{1} \in A_{1}$, which contradicts the definition of $r_{1}$. The proof of the second equation (with $a_{2}$ and $r_{2}$ ) is the same.

2. Being straight rays emanating from 0 , the images of the curves $\Gamma_{\alpha_{D}}\left(r_{1}, \cdot\right)$ and $\Gamma_{\alpha_{D}}\left(r_{2}, \cdot\right)$ are either identical or have only the point 0 in common. Therefore, regarding their lifts we deduce that if $\Gamma_{\alpha_{D}}^{f, a_{1}}\left(r_{1}, t_{1}\right)=$ $\Gamma_{\alpha_{D}}^{f, a_{2}}\left(r_{2}, t_{2}\right)$ and not $t_{1}=t_{2}=0$ then $\operatorname{im} \Gamma_{\alpha_{D}}^{f, a_{1}}\left(r_{1}, \cdot\right)=\operatorname{im} \Gamma_{\alpha_{D}}^{f, a_{2}}\left(r_{2}, \cdot\right)$.

Thus, if assertion 2 were not valid we could conclude (following the curves backwards if necessary) that either $\alpha_{D}\left(a_{1}\right) \in \Gamma_{\alpha_{D}}^{f, a_{2}}\left(\left\{r_{2}\right\} \times\left[b_{2}, d_{2}[)\right.\right.$ or $\alpha_{D}\left(a_{2}\right) \in \Gamma_{\alpha_{D}}^{f, a_{1}}\left(\left\{r_{1}\right\} \times\left[b_{1}, d_{1}[)\right.\right.$. However, this contradicts assertion 1 , which is already proven.

3. From Lemma 5.5 and 5.3 we conclude that $b(s)<1$ for all $s \in] v_{k}, r_{1}[$ $(s \in] r_{2}, v_{k+1}\left[\right.$, resp.) Now, the definition of $b_{1}, b_{2}$ implies the assertion. 
Definition 5 (See Figure 1). We define the curve $\gamma_{0} \in C^{0}\left(I_{0} \cup[0,1] \cup\right.$ $\left.I_{1}, \mathbb{R}^{2}\right)$ by

$$
\gamma_{0}(s):= \begin{cases}\Gamma_{\alpha_{D}}^{f, a_{1}}\left(r_{1}, b_{1}-s\right) & \text { if } s \in I_{1}, \\ \alpha_{D}\left(a_{1}(1-s)+a_{2} s\right) & \text { if } s \in[0,1], \\ \Gamma_{\alpha_{D}}^{f, a_{2}}\left(r_{2}, b_{2}+s-1\right) & \text { if } s \in I_{2} .\end{cases}
$$

Here, $I_{1}$ and $I_{2}$ denote the following intervals:

$$
\begin{aligned}
& \left.\left.\left.\left.I_{1}:=\{s \in]-\infty, 0\right] \mid b_{1}-s \in \Omega_{\alpha_{D}}^{a_{1}}\left(r_{1}\right)\right\}=\right] b_{1}-d_{1}, 0\right], \\
& I_{2}:=\left\{s \in \left[1, \infty\left[\mid b_{2}+s-1 \in \Omega_{\alpha_{D}}^{a_{2}}\left(r_{2}\right)\right\}=\left[1, d_{2}-b_{2}+1[.\right.\right.\right.
\end{aligned}
$$

Of course, the maximum of $I_{1}$ is 0 and the minimum of $I_{2}$ is 1 .

LEMMA 9. 1. $\gamma_{0}$ is injective.

2. $d_{1} \leq 1$, i.e. $\left.\left.I_{1} \subset\right] b_{1}-1,0\right]$, and $d_{2} \leq 1$, i.e. $I_{2} \subset\left[1,2-b_{2}[\right.$. In particular, $b_{1}, b_{2}<1$ (since $b_{1} \in \Omega_{\alpha_{D}}^{a_{1}}\left(r_{1}\right)$ and $\left.b_{2} \in \Omega_{\alpha_{D}}^{a_{2}}\left(r_{2}\right)\right)$.

Pro of. 1. This is a direct consequence of Definition\&Lemma 7.1.

2. Assume that $d_{1}>1$. Definition\&Lemma 7.3 shows that $b_{1} \leq 1$. Therefore, $\Gamma_{\alpha_{D}}^{f, a_{1}}\left(r_{1}, \cdot\right)$ is defined at 1 and Definition\&Lemma 7.1 shows that $\Gamma_{\alpha_{D}}^{f, a_{1}}\left(r_{1}, 1\right) \neq \alpha_{D}\left(r_{1}\right)$. By continuity we conclude that also $\Gamma_{\alpha_{D}}^{f, a\left(r_{1}^{n}\right)}\left(r_{1}^{n}, 1\right) \neq$ $\alpha_{D}\left(r_{1}^{n}\right)$ for all large enough $n$.

At the same time we know that $\Gamma_{\alpha_{D}}^{f}\left(r_{1}^{n}, \cdot\right)=\Gamma_{\alpha_{D}}^{f, a\left(r_{1}^{n}\right)}\left(r_{1}^{n}, \cdot\right)$ since $r_{1}^{n} \in A_{1}$. However, this yields a contradiction since $\Gamma_{\alpha_{D}}^{f}\left(r_{1}^{n}, 1\right)=\alpha_{D}\left(r_{1}^{n}\right)$ by definition.

The proof for $d_{2}$ is the same.

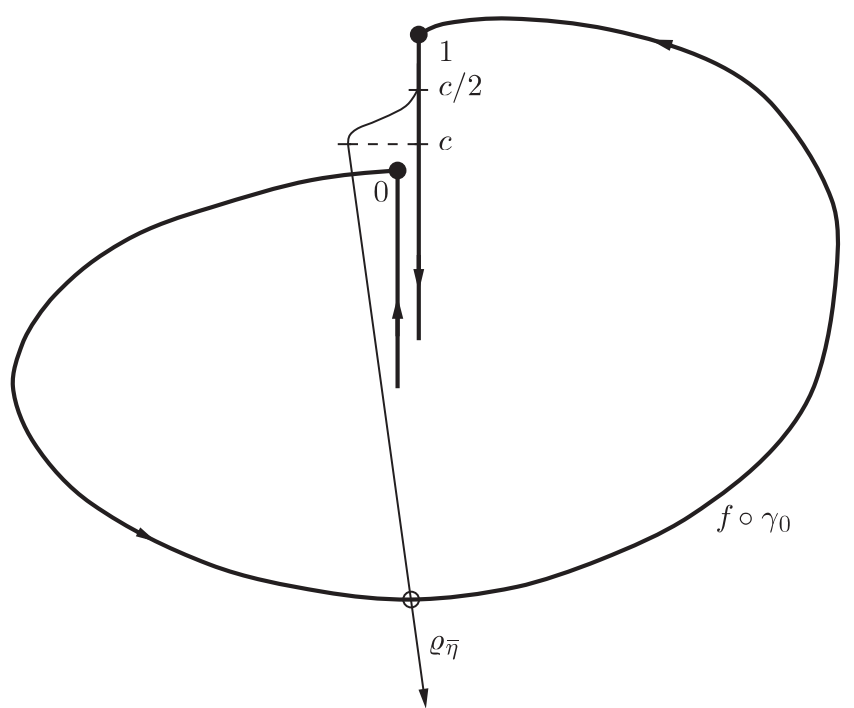

Fig. 2 
Definition\&Lemma 8 (See Figure 2). If $r_{1}<r_{2}$ we take $\gamma:=\gamma_{0}$. If $r_{1}=r_{2}$ we improve $\gamma_{0}$ in the following way:

We set $\omega_{0}:=d_{2}-b_{2}+1$ (i.e. $I_{2}=\left[1, \omega_{0}[)\right.$ and $c:=\left(1+\omega_{0}\right) / 2$. Now we take a $C^{1}$-function $\Psi:\left[1, \infty\left[\rightarrow[0,1]\right.\right.$ with $\Psi^{-1}(0)=[1, c / 2]$ and $\Psi^{-1}(1)=[c, \infty[$. For every $\eta \in] 0, \infty\left[\right.$ we define $\varrho_{\eta}:\left[1, \infty\left[\rightarrow \mathbb{R}^{2}\right.\right.$ by

$$
\varrho_{\eta}(s):=\Gamma_{\alpha_{D}}\left(r_{2}, s+b_{2}-1\right)+\eta\left(s+b_{2}-2\right) \Psi(s) \Gamma_{\alpha_{D}}\left(r_{2}, 1\right)^{\perp},
$$

where $(x, y)^{\perp}:=(-y, x)$. Let $\varrho_{\eta}^{f}$ be the lift of $\varrho_{\eta}$ with respect to $f$ such that $\varrho_{\eta}^{f}(1)=\gamma_{0}(1) \in f^{-1}\left(\varrho_{\eta}(1)\right)$ and let $\left[1, \omega_{\eta}\right.$ [ denote the associated maximal interval of existence. Now we define

$$
\gamma_{\eta}(s):= \begin{cases}\gamma_{0}(s) & \text { if } s \in I_{1} \cup[0,1] \\ \varrho_{\eta}^{f}(s) & \text { if } s \in\left[1, \omega_{\eta}[.\right.\end{cases}
$$

Then there is a $\bar{\eta}>0$ such that the curve

$$
\gamma:= \begin{cases}\gamma_{0} \circ \beta & \text { if } r_{1}<r_{2}, \\ \gamma_{\bar{\eta}} \circ \beta & \text { if } r_{1}=r_{2},\end{cases}
$$

where $\beta: I_{1} \cup[0,1] \cup\left[1, \omega_{\bar{\eta}}\left[\rightarrow I_{1} \cup[0,1] \cup I_{2}\right.\right.$ is an orientation preserving and regular reparametrization with $\beta(0)=0, \beta(1)=c$, has the following properties:

1. $\gamma$ is injective.

2. $\gamma$ is proper and p.w. regular.

3. There is an $\varepsilon>0$ such that

$$
\angle(f \circ \gamma)^{\cdot}\left(s_{2}\right)-\angle(f \circ \gamma)^{\cdot}\left(s_{1}\right) \geq 3 \pi+\varepsilon \quad \text { for all } s_{2} \in I_{2} \backslash\{1\}, s_{1} \in I_{1} \backslash\{0\} \text {. }
$$

Proof. 1. If $r_{1}<r_{2}$ the assertion follows from Lemma 9.1. So let us consider the case $r_{1}=r_{2}$. Since $\varrho_{\eta}$ is injective by definition we conclude that $\gamma_{\eta} \mid\left[1, \omega_{\eta}\left[=\varrho_{\eta}^{f}\right.\right.$ is also injective. From Lemma 9.1 we know that so is $\gamma_{\eta}\left|I_{1} \cup[0, c / 2]=\gamma_{0}\right| I_{1} \cup[0, c / 2]$. Thus, in order to prove the injectivity of $\gamma_{\eta}$ for some $\eta>0$ it remains to show that $\left.\varrho_{\eta}^{f} \mid\right] c / 2, \omega_{\eta}$ [ cannot intersect $\gamma_{0} \mid I_{1} \cup[0,1]:$

The definition of $\varrho_{\eta}$ shows that for all $\eta>0$ the only intersection of $\left.\varrho_{\eta} \mid\right] c / 2, \infty\left[\right.$ and $\Gamma_{\alpha_{D}}\left(r_{2}, \cdot\right)=\Gamma_{\alpha_{D}}\left(r_{1}, \cdot\right)$ is

$$
\varrho_{\eta}\left(2-b_{2}\right)=\Gamma_{\alpha_{D}}\left(r_{2}, 1\right) \text {. }
$$

However, the point $\Gamma_{\alpha_{D}}\left(r_{2}, 1\right)$ is neither lifted to $\Gamma_{\alpha_{D}}^{f, a_{1}}\left(r_{1}, \cdot\right)$ nor to $\Gamma_{\alpha_{D}}^{f, a_{2}}\left(r_{2}, \cdot\right)$ since $1 \notin \Omega_{\alpha_{D}}^{a_{1}}\left(r_{1}\right)$ and $1 \notin \Omega_{\alpha_{D}}^{a_{2}}\left(r_{2}\right)$ (see Lemma 9.2). Using Definition 5 this shows that $\left.\varrho_{\eta}^{f} \mid\right] c / 2, \omega_{\eta}$ [ cannot intersect $\gamma_{0} \mid I_{1}$ or $\gamma_{0} \mid I_{2}$. Thus we are left with proving that $\left.\varrho_{\eta}^{f} \mid\right] c / 2, \omega_{\eta}$ [ cannot intersect $\left.\gamma_{0} \mid\right] 0,1[$ :

Let us assume the contrary and let $\varrho_{\eta}^{f}\left(q_{\eta}\right)=\gamma_{0}\left(p_{\eta}\right)$ denote the first intersection. Then the injectivity of $\varrho_{\eta}^{f}$ and $\gamma_{0}$ implies that $\gamma_{\eta} \mid\left[p_{\eta}, q_{\eta}\right]$ is a closed Jordan curve dividing $\mathbb{R}^{2}$ into two components. We conclude that the bounded interior component $G_{c}$ is on the right side of $\gamma_{\eta} \mid\left[p_{\eta}, q_{\eta}\right]$ : Indeed, 
$\gamma_{0} \mid I_{2}$ branches off $\varrho_{\eta}^{f}$ to the left side (see the definition of $\varrho_{\eta}^{f}$ together with Definition 5).

Since we already know that $\gamma_{0} \mid I_{2}$ and $\left.\varrho_{\eta}^{f} \mid\right] c / 2, \omega_{\eta}$ [ cannot intersect and since $\gamma_{0}$ is injective we conclude that $\gamma_{0}$ cannot cross $\partial G_{c}=\gamma_{0}\left(\left[p_{\eta}, c / 2\right]\right) \cup$ $\varrho_{\eta}^{f}\left(\left[c / 2, q_{\eta}\right]\right)$. Therefore, if $G_{c}$ were on the left side of $\gamma_{\eta} \mid\left[p_{\eta}, q_{\eta}\right]$ then $\gamma_{0}\left(I_{2}\right)$ would be contained in $G_{c}$, which is impossible since $\gamma_{0}$ is proper and therefore $\gamma_{0}\left(I_{2}\right)$ is unbounded.

Thus we conclude that $\gamma_{\eta} \mid\left[p_{\eta}, q_{\eta}\right]$ is a right-oriented Jordan curve, which implies that the total rotation of the tangent angle is $-2 \pi$. Since $f$ is orientation preserving the same is true for $f \circ \gamma_{\eta} \mid\left[p_{\eta}, q_{\eta}\right]$. (This can be shown by shrinking the curve (apply an isotopy $[0,1] \times \mathbb{R}^{2} \rightarrow \mathbb{R}^{2}$ ) into a neighbourhood of zero where $f$ is a diffeomorphism.)

More precisely, this means that

$$
\begin{aligned}
\angle\left(f \circ \gamma_{0}\right)^{\cdot}(1)-\angle\left(f \circ \gamma_{0}\right)^{\cdot} & \left(p_{\eta}\right)+\Delta_{\eta}^{1} \\
& +\angle\left(f \circ \varrho_{\eta}^{f}\right)^{\cdot}\left(q_{\eta}\right)-\angle\left(f \circ \varrho_{\eta}^{f}\right)^{\cdot}(1)+\Delta_{\eta}^{p, q}=-2 \pi
\end{aligned}
$$

with $\Delta_{\eta}^{1}, \Delta_{\eta}^{p, q}$ denoting the tangent angle jumps of the two edges at $\gamma_{\eta}(1)$ and $\gamma_{\eta}\left(p_{\eta}\right)=\gamma_{\eta}\left(q_{\eta}\right)$. Therefore,

$$
\Delta_{\eta}^{1}, \Delta_{\eta}^{p, q} \in[-\pi, \pi] .
$$

Thus we estimate

$$
\angle\left(f \circ \gamma_{0}\right)^{\cdot}(1)-\angle\left(f \circ \gamma_{0}\right)^{\cdot}\left(p_{\eta}\right) \leq \angle\left(f \circ \varrho_{\eta}^{f}\right)^{\cdot}\left(q_{\eta}\right)-\angle\left(f \circ \varrho_{\eta}^{f}\right)^{\cdot}(1) .
$$

Now, we assume that assertion 1 is false. Then there is a sequence $\eta_{k} \rightarrow 0$ such that $p_{\eta_{k}}, q_{\eta_{k}}$ exist for all $k \in \mathbb{N}$ (i.e. $\left.\varrho_{\eta_{k}}^{f} \mid\right] c / 2, \omega_{\eta_{k}}$ [intersects $\left.\gamma_{0} \mid\right] 0,1[$ ).

Since $\left.p_{\eta_{k}} \in\right] 0,1\left[\right.$ we conclude that $q_{\eta_{k}}$ also remains bounded. Therefore, passing to a subsequence if necessary, we may assume that $p_{\eta_{k}} \rightarrow p_{0} \in[0,1]$ and $q_{\eta_{k}} \rightarrow q_{0} \in\left[c / 2, \infty\left[\right.\right.$. Clearly, the definition of $\varrho_{\eta}$ shows that

$$
f \circ \gamma_{0}\left(p_{0}\right)=f \circ \varrho_{0}^{f}\left(q_{0}\right) \text {. }
$$

Since $f \circ \varrho_{0}^{f}=\varrho_{0} \mid\left[1, \omega_{0}\left[\right.\right.$ is injective and $q_{0} \neq 1$ it follows that $f \circ \varrho_{0}^{f}\left(q_{0}\right) \neq$ $f \circ \varrho_{0}^{f}(1)=\varrho_{0}(1)=f \circ \gamma_{0}(1)$ and thus we conclude that $p_{0} \neq 1$, i.e. $p_{0} \in[0,1[$.

Taking into account that $f \circ \varrho_{\eta}^{f}=\varrho_{\eta}$ it follows from the definition of $\varrho_{\eta}$ that the right-hand side of inequality (8) converges to 0 as $\eta \rightarrow 0$. Thus we conclude that

$$
\angle\left(f \circ \gamma_{0}\right)^{\cdot}(1)-\angle\left(f \circ \gamma_{0}\right)^{\cdot}\left(p_{0}\right) \leq 0 .
$$

By using Definition 5 this may be reformulated as

$$
\angle\left(f \circ \alpha_{D}\right)^{\cdot}\left(a_{2}\right)-\angle\left(f \circ \alpha_{D}\right)^{\cdot}\left(\widetilde{p}_{0}\right) \leq 0
$$

with a $\widetilde{p}_{0} \in\left[a_{1}, a_{2}[\right.$. In the same way equation (9) translates to

$$
f \circ \alpha_{D}\left(\widetilde{p}_{0}\right)=\Gamma_{\alpha_{D}}\left(r_{2}, \widetilde{q}_{0}\right)
$$

with a $\left.\widetilde{q}_{0} \in\right] b_{2}, \infty\left[\right.$. Since $\widetilde{q}_{0}>0$ this implies that $\Gamma_{\alpha_{D}}\left(r_{2}, \widetilde{q}_{0}\right) \neq 0$ and 
therefore that (we use Definition 2.4)

$$
\angle f \circ \alpha_{D}\left(\widetilde{p}_{0}\right)=\angle \Gamma_{\alpha_{D}}\left(r_{2}, \widetilde{q}_{0}\right)=\angle f \circ \alpha_{D}\left(r_{2}\right) \bmod 2 \pi .
$$

Now we have to distinguish three cases:

Case 1: $\left.\widetilde{p}_{0} \in\right] v_{k}, v_{k+1}\left[\right.$. Since $\gamma_{0}$ is proper and injective it divides $\mathbb{R}^{2}$ into two components, one being to the right of $\gamma_{0}$ which we will call $G_{r}$ and the other, $G_{l}$, being to the left. As already previously used in this proof, $\varrho_{\eta_{k}}^{f}$ branches off $\gamma_{0}$ to the right side (obvious from the definition of $\varrho_{\eta}^{f}$ together with Definition 5). Therefore, $\varrho_{\eta_{k}}^{f}(] c / 2, q_{\eta_{k}}[) \subset G_{r}$ and the first intersection of $\left.\varrho_{\eta_{k}}^{f} \mid\right] c / 2, \omega_{\eta_{k}}$ [ with $\gamma_{0}$ at $q_{\eta_{k}}$ must be from right to left. Since $f$ is orientation preserving we conclude that $\Delta_{\eta_{k}}^{p, q} \in[-\pi, 0]$. Using the fact that clearly $\Delta_{\eta_{k}}^{p, q}=\angle\left(f \circ \gamma_{0}\right)^{\cdot}\left(p_{\eta_{k}}\right)-\angle\left(f \circ \varrho_{\eta_{k}}^{f}\right)^{\cdot}\left(q_{\eta_{k}}\right)$ and also some redefinitions (Definition\&Lemma 2.2, Definition 2.4, Definition 5 and the definition of $\left.\varrho_{\eta}\right)$ it follows that $\Theta_{\alpha_{D}}\left(\widetilde{p}_{0}\right) \bmod 2 \pi=\lim _{k \rightarrow \infty} \Delta_{\eta_{k}}^{p, q} \in[-\pi, 0]$ by continuity. However, Lemma 7 together with Lemma 2 shows that $\Theta_{\alpha_{D}}\left(\widetilde{p}_{0}\right) \bmod 2 \pi \in$ ] $0, \pi[$, a contradiction.

Therefore, case 1 cannot occur.

In order to treat cases 2 and 3 we will frequently use the fact that $\angle f \circ \alpha_{D}$ is strictly decreasing on $\left[a_{1}, v_{k}\right]$ and on $\left[v_{k+1}, a_{2}\right]$ (DEC) and is strictly increasing on $\left[v_{k}, v_{k+1}\right]$ (INC) (Lemmas 7 and 2). In addition, we need the following:

Lemma 5.6 shows that $\angle f \circ \alpha_{D} \circ a\left(r_{1}^{n}\right) \leq \angle f \circ \alpha_{D}\left(r_{1}^{n}\right)\left(\angle f \circ \alpha_{D} \circ a\left(r_{2}^{n}\right) \geq\right.$ $\angle f \circ \alpha_{D}\left(r_{2}^{n}\right)$, resp.) for all $n \in \mathbb{N}$. Thus, passing to the limit we deduce that

$$
\angle f \circ \alpha_{D}\left(a_{1}\right) \leq \angle f \circ \alpha_{D}\left(r_{1}\right), \quad \angle f \circ \alpha_{D}\left(a_{2}\right) \geq \angle f \circ \alpha_{D}\left(r_{2}\right)
$$

by continuity.

Case 2: $\widetilde{p}_{0} \in\left[v_{k+1}, a_{2}\left[\right.\right.$. Lemma 6 shows that $\mid \angle f \circ \alpha_{D}\left(r_{2}^{n}\right)-\angle f \circ$ $\alpha_{D}\left(v_{k+1}\right) \mid \leq \pi$ for all $n \in \mathbb{N}$. Thus, passing to the limit we conclude that

$$
\left|\angle f \circ \alpha_{D}\left(r_{2}\right)-\angle f \circ \alpha_{D}\left(v_{k+1}\right)\right| \leq \pi .
$$

From (DEC) and inequality (12) it follows that $\angle f \circ \alpha_{D}\left(\widetilde{p}_{0}\right)>\angle f \circ \alpha_{D}\left(a_{2}\right) \geq$ $\angle f \circ \alpha_{D}\left(r_{2}\right)$. Using equation (11) this implies that

$$
\angle f \circ \alpha_{D}\left(\widetilde{p}_{0}\right) \geq \angle f \circ \alpha_{D}\left(r_{2}\right)+2 \pi .
$$

Since $\angle f \circ \alpha_{D}\left(v_{k+1}\right) \geq \angle f \circ \alpha_{D}\left(\widetilde{p}_{0}\right)(\mathrm{DEC})$ this leads to

$$
\angle f \circ \alpha_{D}\left(v_{k+1}\right) \geq \angle f \circ \alpha_{D}\left(r_{2}\right)+2 \pi,
$$

which contradicts inequality (13). Therefore, case 2 cannot occur either.

Case 3: $\widetilde{p}_{0} \in\left[a_{1}, v_{k}\right]$. From (DEC), (INC), inequality (12) and Definition\&Lemma 7 it follows that

$$
\begin{aligned}
\angle f \circ \alpha_{D}\left(\widetilde{p}_{0}\right) & \leq \angle f \circ \alpha_{D}\left(a_{1}\right) \leq \angle f \circ \alpha_{D}\left(r_{1}\right) \\
& \leq \angle f \circ \alpha_{D}\left(r_{2}\right) \leq \angle f \circ \alpha_{D}\left(a_{2}\right) .
\end{aligned}
$$


By Lemma 7 and since $v_{k}, v_{k+1}$ are transversal (see Definition\&Lemma 3) we may also estimate

$$
\Theta_{\alpha_{D}}\left(a_{2}\right)-\Theta_{\alpha_{D}}\left(\widetilde{p}_{0}\right) \geq \Theta_{\alpha_{D}}\left(v_{k+1}\right)-\Theta_{\alpha_{D}}\left(v_{k}\right)=\pi .
$$

Thus, using the definition of $\Theta_{\alpha_{D}}$ (see Definition\&Lemma 2) together with (14) and (15) we calculate

$$
\begin{aligned}
& \angle\left(f \circ \alpha_{D}\right)^{\cdot}\left(a_{2}\right)-\angle\left(f \circ \alpha_{D}\right)^{\cdot}\left(\widetilde{p}_{0}\right) \\
& \quad=\angle f \circ \alpha_{D}\left(a_{2}\right)-\angle f \circ \alpha_{D}\left(\widetilde{p}_{0}\right)+\Theta_{\alpha_{D}}\left(a_{2}\right)-\Theta_{\alpha_{D}}\left(\widetilde{p}_{0}\right) \geq \pi,
\end{aligned}
$$

which contradicts inequality (10).

This shows that case 3 is also impossible.

Therefore, there is an $\eta_{0}>0$ such that $\gamma_{\eta}$ is injective for all $\eta$ with $0<\eta \leq \eta_{0}$.

However, in order to make it possible to prove assertion 3 we still have to improve this $\eta$ :

Since $f \circ \gamma_{0} \mid I_{2}$ is injective, so is the restriction $f \mid \gamma_{0}([1, c])$. Now, the same argument as in Lemma 4.3 shows that this injectivity of $f$ even extends to a neighbourhood $U$ of $\gamma_{0}([1, c])$, i.e. $f \mid U: U \rightarrow f(U)$ is a diffeomorphism. Clearly $f(U)$ is a neighbourhood of $f \circ \gamma_{0}([1, c])$.

Since $f \circ \gamma_{\eta}\left|[1, c] \rightarrow f \circ \gamma_{0}\right|[1, c]$ uniformly as $\eta \rightarrow 0$, we can choose an $\bar{\eta}$ such that $f \circ \gamma_{\bar{\eta}}([1, c]) \subset f(U)$ and $0<\bar{\eta} \leq \eta_{0}$. Since $\gamma_{\bar{\eta}}(1)=\gamma_{0}(1) \in U$ this ensures that

$$
\omega_{\bar{\eta}}>c
$$

2. Obvious.

3. A straightforward calculation using Lemma 7 shows that

$$
\angle\left(f \circ \gamma_{0}\right)^{\cdot}\left(s_{2}\right)-\angle\left(f \circ \gamma_{0}\right)^{\cdot}\left(s_{1}\right)=3 \pi+f \circ \alpha_{D}\left(r_{2}\right)-f \circ \alpha_{D}\left(r_{1}\right)
$$

for all $s_{2} \in I_{2} \backslash\{1\}, s_{1} \in I_{1} \backslash\{0\}$. From Lemmas 2 and 7 we know that $\varepsilon:=f \circ \alpha_{D}\left(r_{2}\right)-f \circ \alpha_{D}\left(r_{1}\right)>0$ if $r_{2}>r_{1}$, which proves the assertion in this case.

If $r_{1}=r_{2}$ we calculate for all $s \in\left[c, \omega_{\bar{\eta}}[\neq \emptyset\right.$ (see (16)) that

$$
\begin{aligned}
\angle\left(f \circ \gamma_{\bar{\eta}}\right)^{\cdot}(s) & =\angle \dot{\varrho}_{\bar{\eta}}(s)=f \circ \alpha_{D}\left(r_{2}\right)+\bar{\eta} f \circ \alpha_{D}\left(r_{2}\right)^{\perp} \\
& =\angle\left(f \circ \gamma_{0}\right)^{\cdot}(s)+\arctan \bar{\eta} .
\end{aligned}
$$

This proves the assertion in the case $r_{1}=r_{2}$ with $\varepsilon:=\arctan \bar{\eta}$.

In order to make use of hypothesis 2 of Theorem 1 we now shift our curve $\gamma$ completely into the region where hypothesis 2 holds, i.e. into $\mathbb{R}^{2} \backslash K$. More precisely, we have the following

Lemma 10. There is a curve $\bar{\gamma} \in C^{0}\left(I_{1} \cup[0,1] \cup I_{2}, \mathbb{R}^{2}\right)$ with the following properties:

1. $\operatorname{im} \bar{\gamma} \subset \mathbb{R}^{2} \backslash K$. 
2. $\bar{\gamma}$ is injective, proper and p.w. regular.

3. There is an $\varepsilon>0$ such that

$$
\angle(f \circ \bar{\gamma})^{\cdot}\left(s_{2}\right)-\angle(f \circ \bar{\gamma})^{\cdot}\left(s_{1}\right) \geq 3 \pi+\varepsilon
$$

for all $s_{2} \in \bar{I}_{2} \backslash\{1\}, s_{1} \in \bar{I}_{1} \backslash\{0\}$ with $\left.\left.\bar{I}_{1}:=I_{1} \cap\right]-\infty, \bar{s}_{1}\right]$ and $\bar{I}_{2}:=I_{2} \cap$ $\left[\bar{s}_{2}, \infty\left[\right.\right.$ for some $\bar{s}_{1} \in I_{1}$ and $\bar{s}_{2} \in I_{2}$.

Proof. In order to shift $\gamma$ out of $K$ we first construct a suitable diffeotopy:

Since $K$ is compact and $\gamma$ is proper and injective we find a $p \in \mathbb{R}^{2} \backslash \operatorname{im} \gamma$ such that

$$
c:=\min \left\{\|\gamma(s)-p\|_{2} \mid s \in I_{1} \cup[0,1] \cup I_{2}\right\}
$$

and

$$
d:=2 \max \left\{\|x-p\|_{2} \mid x \in K\right\}
$$

exist. Of course, $c>0$.

Now we choose an auxiliary function $\varrho \in C^{1}\left(\left[0, \infty\left[,\left[0, \infty[)\right.\right.\right.\right.$ with $\varrho^{\prime}(s)>0$ everywhere, $\varrho(c)=d$ and $\varrho(s)=s$ if $s \geq 2 d$ or $s=0$. (It is easy to see that such functions exist.) This enables us to define the diffeotopy $h \in$ $C^{1}\left([0,1] \times \mathbb{R}^{2}, \mathbb{R}^{2}\right)$ by

$$
h(t, x):= \begin{cases}\frac{\left((1-t)\|x-p\|_{2}+t \varrho\left(\|x-p\|_{2}\right)\right)(x-p)}{\|x-p\|_{2}}+p & \text { if } x \neq p, \\ p & \text { if } x=p .\end{cases}
$$

Obviously, $h$ inherits from $\varrho$ the following properties:

(i) $D_{x} h$ is invertible everywhere and $h(t, \cdot)$ is a diffeomorphism for every $t \in[0,1]$.

(ii) $\|h(1, x)-p\|_{2} \geq d$ if $\|x-p\|_{2} \geq c$,

(iii) $h(t, x)=x$ if $t=0$ or $\|x-p\|_{2} \geq 2 d$ or $x=p$.

Now we define $\bar{\gamma}(s):=h(1, \gamma(s))$. Then assertion 1 is obvious from (ii). Assertion 2 follows from (i), (iii) and (i), resp. together with Definition \&Lemma 8.1, 2.

Assertion 3: Since $\gamma$ is proper,

$$
\begin{aligned}
& \bar{s}_{1}:=\max \left\{s \in I_{1} \mid\left\|\gamma\left(s^{\prime}\right)-p\right\|_{2} \geq 2 d \text { for all } s^{\prime} \in I_{1}, s^{\prime} \leq s\right\}, \\
& \bar{s}_{2}:=\min \left\{s \in I_{2} \mid\left\|\gamma\left(s^{\prime}\right)-p\right\|_{2} \geq 2 d \text { for all } s^{\prime} \in I_{2}, s^{\prime} \geq s\right\}
\end{aligned}
$$

exist and we define $\left.\left.\bar{I}_{1}:=I_{1} \cap\right]-\infty, \bar{s}_{1}\right]$ and $\bar{I}_{2}:=I_{2} \cap\left[\bar{s}_{2}, \infty[\right.$. Then (iii) shows that for all $s_{1} \in \bar{I}_{1} \backslash\{0\}, s_{2} \in \bar{I}_{2} \backslash\{1\}$ and $t \in[0,1]$,

$$
\begin{aligned}
& \angle(f \circ h(t, \gamma(\cdot)))^{\cdot}\left(s_{1}\right) \bmod 2 \pi=\angle(f \circ \gamma)^{\cdot}\left(s_{1}\right) \bmod 2 \pi, \\
& \angle(f \circ h(t, \gamma(\cdot)))^{\cdot}\left(s_{2}\right) \bmod 2 \pi=\angle(f \circ \gamma)^{\cdot}\left(s_{2}\right) \bmod 2 \pi
\end{aligned}
$$


since $\bar{\gamma}\left|\bar{I}_{1}=\gamma\right| \bar{I}_{1}$ and $\bar{\gamma}\left|\bar{I}_{2}=\gamma\right| \bar{I}_{2}$. Moreover, the angle $\angle(f \circ h(t, \gamma(\cdot)))^{\cdot}(s)$ is defined everywhere (by (i)) and it is a continuous function of $t$ and $s$. Therefore the two equations remain valid even if the $\bmod 2 \pi$ term is dropped and the assertion follows from Definition\&Lemma 8.3.

Since Lemma 10 is a central result about self-immersions of the plane we restate it as a theorem using an obvious reparametrization and a smoothing of the two edges of the curve:

TheOREM 2. Let $f \in C^{1}\left(\mathbb{R}^{2}, \mathbb{R}^{2}\right)$ be not injective and such that $\operatorname{det} D f(x)>0$ for all $x \in \mathbb{R}^{2}$. Then for every compact set $K$ there is a curve $\gamma \in C^{1}\left(\mathbb{R}, \mathbb{R}^{2} \backslash K\right)$ with the following properties:

1. $\gamma$ is injective, proper and regular.

2. There is an $\varepsilon>0$ such that for every $s_{1} \leq 0$ and $s_{2} \geq 1$ the rotation of $(f \circ \gamma)^{\cdot}$ from $s_{1}$ to $s_{2}$ is at least $3 \pi+\varepsilon$.

Next, we will prove a general property of certain curves in the plane. This result is completely independent of the map $f$.

Theorem 3. Let $\gamma \in C^{1}\left(\mathbb{R}, \mathbb{R}^{2}\right)$ be injective, proper and regular. Then for every $\varepsilon>0$ there are $s_{1} \leq 0$ and $s_{2} \geq 1$ such that the rotation of $\dot{\gamma}(s)$ from $s_{1}$ to $s_{2}$ is less than $\pi+\varepsilon$.

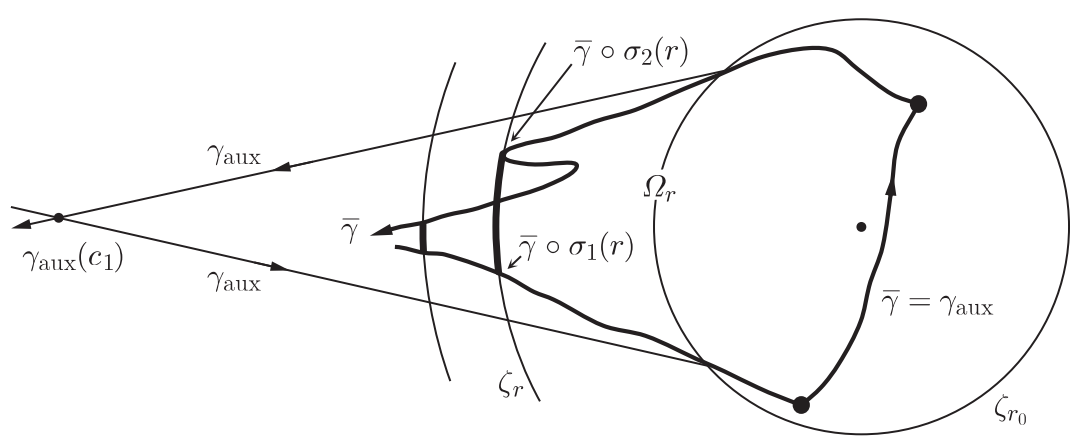

Fig. 3

Pro of (See Figure 3). We assume the contrary, i.e. there is an $\varepsilon>0$ such that

$$
\angle \dot{\gamma}\left(s_{2}\right)-\angle \dot{\gamma}\left(s_{1}\right) \geq \pi+\varepsilon \quad \text { for all } s_{1} \leq 0, s_{2} \geq 1 .
$$

Let $R:=\max \gamma([0,1])+1$. Then, for every $r \geq R$ we define the last intersection $\sigma_{1}(r)$ of $\left.\left.\gamma \mid\right]-\infty, 0\right]$ with the circle $\left\{x \in \mathbb{R}^{2} \mid\|x\|_{2}=r\right\}$ by

$$
\left.\left.\sigma_{1}(r):=\max \{s \in]-\infty, 0\right] \mid\|\gamma(s)\|_{2}=r\right\}
$$

and also the first intersection $\sigma_{2}(r)$ of $\gamma \mid[0, \infty[$ with the same circle by

$$
\sigma_{2}(r):=\min \left\{s \in \left[0, \infty\left[\mid\|\gamma(s)\|_{2}=r\right\} .\right.\right.
$$


(Notice that $\sigma_{1}(r), \sigma_{2}(r)$ always exist since $\gamma$ is proper.)

For every $r \geq R$ let $\zeta_{r}$ be a regular left-oriented (i.e. such that the interior is to the left) parametrization of the circle $\left\{x \in \mathbb{R}^{2} \mid\|x\|_{2}=r\right\}$ over $\left[\sigma_{2}(r), \sigma_{2}(r)+2 \pi\right]$ such that $\zeta_{r}\left(\sigma_{2}(r)\right)=\gamma\left(\sigma_{2}(r)\right)$ and $\zeta_{r}\left(\sigma_{2}(r)+1\right)=$ $\gamma\left(\sigma_{1}(r)\right)$.

Let $\iota_{r}:\left[\sigma_{1}(r), \sigma_{2}(r)+1\right] \rightarrow \mathbb{R}^{2}$ be the p.w. regular closed Jordan curve ( $\gamma$ is injective!)

$$
\iota_{r}(s):= \begin{cases}\gamma(s) & \text { if } s \in\left[\sigma_{1}(r), \sigma_{2}(r)\right], \\ \zeta_{r}(s) & \text { if } s \in\left[\sigma_{2}(r), \sigma_{2}(r)+1\right],\end{cases}
$$

and let $\Omega_{r}$ denote its open interior region.

We also need the rotation of the tangent angle at the two "edges" of $\iota_{r}$ :

$$
\begin{aligned}
& \varphi_{1}(r):=\left(\angle \dot{\gamma}\left(\sigma_{1}(r)\right)-\angle \dot{\zeta}_{r}\left(\sigma_{2}(r)+1\right)\right) \bmod 2 \pi \in[0,2 \pi[, \\
& \varphi_{2}(r):=\left(L \dot{\zeta}_{r}\left(\sigma_{2}(r)\right)-\angle \dot{\gamma}\left(\sigma_{2}(r)\right)\right) \bmod 2 \pi \in[0,2 \pi[.
\end{aligned}
$$

Moreover, we use the abbreviations

$$
\begin{aligned}
& \varphi_{\gamma}(r):=\angle \dot{\gamma}\left(\sigma_{2}(r)\right)-\angle \dot{\gamma}\left(\sigma_{1}(r)\right), \\
& \varphi_{\zeta}(r):=\angle \dot{\zeta}_{r}\left(\sigma_{2}(r)+1\right)-\angle \dot{\zeta}_{r}\left(\sigma_{2}(r)\right) .
\end{aligned}
$$

Our assumption (17) shows that

$$
\varphi_{\gamma}(r) \geq \pi+\varepsilon
$$

and since $\zeta_{r}$ is left-oriented it follows that

$$
0<\varphi_{\zeta}(r)<2 \pi .
$$

Observing that $\gamma$ enters $\Omega_{r}$ at $\sigma_{1}(r)$ and leaves $\Omega_{r}$ at $\sigma_{2}(r)$ it is clear that

$$
0 \leq \varphi_{i}(r) \leq \pi \quad(i=1,2) .
$$

Since $\iota_{r}$ is a closed Jordan curve the total rotation of its tangent angle must be either $2 \pi$ or $-2 \pi$, i.e.

$$
\varphi_{\gamma}(r)+\varphi_{2}(r)+\varphi_{\zeta}(r)+\varphi_{1}(r)= \pm 2 \pi .
$$

An easy calculation using the statements (18)-(21) yields

$$
\begin{gathered}
\varphi_{\gamma}(r)+\varphi_{2}(r)+\varphi_{\zeta}(r)+\varphi_{1}(r)=2 \pi \\
0 \leq \varphi_{i}(r)<\pi-\varepsilon \quad(i=1,2), \\
0<\varphi_{\zeta}(r) \leq \pi-\varepsilon .
\end{gathered}
$$

In addition equation (22) shows that $\Omega_{r}$ has to be to the left of $\iota_{r}$. Since our assumption (17) shows that $\angle \dot{\gamma}\left(\sigma_{2}(r)\right) \geq \angle \dot{\gamma}\left(\sigma_{1}(R)\right)+\pi+\varepsilon$ for all $r \geq R$ we conclude that $\bar{\varphi}:=\inf _{r \geq R} \angle \dot{\gamma}\left(\sigma_{2}(r)\right)$ exists. Moreover, we find an $r_{0} \geq R$ such that

$$
L \dot{\gamma}\left(\sigma_{2}\left(r_{0}\right)\right) \leq \bar{\varphi}+\varepsilon / 8 .
$$


We also define

$$
\begin{aligned}
& \Phi_{1}:=\bar{\varphi}-(\pi+\varepsilon)+\varepsilon / 4, \\
& \Phi_{2}:=\bar{\varphi}-\varepsilon / 4 .
\end{aligned}
$$

Now, we will prove the following three statements:

$$
\begin{gathered}
\Phi_{2}-\Phi_{1}=\pi+\varepsilon / 2, \\
\Phi_{2}<\angle \dot{\gamma}\left(\sigma_{2}(r)\right)<\Phi_{2}+\pi \quad \text { if } r \geq r_{0}, \\
\Phi_{1}-\pi<\angle \dot{\gamma}\left(\sigma_{1}(r)\right)<\Phi_{1} \quad \text { if } r \geq r_{0} .
\end{gathered}
$$

Equation (28) is obvious from (26) and (27).

Using the definition of $\bar{\varphi}$ and definition (27) the left inequality in (29) follows. The right inequality in (30) follows from our assumption (17), from (25) and from definition (26).

In order to prove the right inequality in (29) we assume the contrary, namely

$$
\angle \dot{\gamma}\left(\sigma_{2}(r)\right) \geq \Phi_{2}+\pi
$$

From this we conclude that (using in addition (22)-(24))

$$
\begin{aligned}
\angle \dot{\gamma}\left(\sigma_{1}(r)\right) & =\angle \dot{\gamma}\left(\sigma_{2}(r)\right)-\varphi_{\gamma}(r) \\
& =\angle \dot{\gamma}\left(\sigma_{2}(r)\right)-2 \pi+\varphi_{\zeta}(r)+\varphi_{1}(r)+\varphi_{2}(r)>\Phi_{2}-\pi .
\end{aligned}
$$

However, using also (25) and (27) this would imply that

$$
\angle \dot{\gamma}\left(\sigma_{2}\left(r_{0}\right)\right)-\angle \dot{\gamma}\left(\sigma_{1}(r)\right)<\bar{\varphi}+\varepsilon / 8-\left(\Phi_{2}-\pi\right)<\pi+\varepsilon,
$$

which contradicts our assumption (17). Thus (29) is proven.

Similarly, we now assume that $\angle \dot{\gamma}\left(\sigma_{1}(r)\right) \leq \Phi_{1}-\pi$ in order to prove the left inequality in (30). Using (22)-(24) and (28) again this would imply that

$$
\angle \dot{\gamma}\left(\sigma_{2}(r)\right)=\angle \dot{\gamma}\left(\sigma_{1}(r)\right)+\varphi_{\gamma}(r)<\Phi_{2},
$$

which contradicts the left inequality in (29).

Now, we define an auxiliary curve $\gamma_{\text {aux }}$ which equals $\gamma$ in some middle part but has ends which are straight lines with the angles $\Phi_{1}$ and $\Phi_{2}$, resp.:

$$
\gamma_{\text {aux }}(s):= \begin{cases}\gamma\left(\sigma_{2}\left(r_{0}\right)\right)+\left(s-\sigma_{2}\left(r_{0}\right)\right) v_{2} & \text { if } s \in\left[\sigma_{2}\left(r_{0}\right), \infty[,\right. \\ \gamma(s) & \text { if } s \in\left[\sigma_{1}\left(r_{0}\right), \sigma_{2}\left(r_{0}\right)\right], \\ \gamma\left(\sigma_{1}\left(r_{0}\right)\right)+\left(s-\sigma_{1}\left(r_{0}\right)\right) v_{1} & \text { if } \left.s \in]-\infty, \sigma_{1}\left(r_{0}\right)\right] .\end{cases}
$$

Here, $v_{1}, v_{2} \in \mathbb{R}^{2}$ are taken such that

$$
\angle v_{i}=\Phi_{i} \bmod 2 \pi, \quad i=1,2 .
$$

Equation (28) shows that the two "ends" of $\gamma_{\text {aux }}$ intersect, i.e. $\gamma_{\text {aux }}\left(c_{1}\right)=$ $\gamma_{\text {aux }}\left(c_{2}\right)$ with a $c_{1}<\sigma_{1}\left(r_{0}\right)$ and a $c_{2}>\sigma_{2}\left(r_{0}\right)$. Then $\gamma_{\text {aux }} \mid\left[c_{1}, c_{2}\right]$ is clearly a left-oriented closed Jordan curve. Let $\Omega_{\text {aux }}$ denote its open interior region and let $r_{c}:=\left\|\gamma_{\text {aux }}\left(c_{1}\right)\right\|_{2}$. 
Now we prove the following auxiliary assertion: if

$$
r \in\left[r_{0}, r_{c}\left[\text { and } \gamma\left(\left[\sigma_{1}(r), \sigma_{2}(r)\right]\right) \subset \bar{\Omega}_{\text {aux }}\right.\right.
$$

then

$$
\zeta_{r}(] \sigma_{2}(r), \sigma_{2}(r)+1[) \subset \Omega_{\mathrm{aux}}
$$

and

$$
\Omega_{r} \subset \Omega_{\text {aux }} .
$$

First, we need the following two facts:

(i) It is clear from our definitions that $\zeta_{r}$ has exactly two transversal intersections with $\partial \Omega_{\text {aux }}$ for every $r \in\left[r_{0}, r_{c}\left[\right.\right.$. One intersection, say at $t_{1}$, is with $\gamma_{\text {aux }} \mid\left[c_{1}, \sigma_{1}\left(r_{0}\right)\right] \subset \partial \Omega_{\text {aux }}$ where $\zeta_{r}$ leaves $\Omega_{\text {aux }}$ (since this intersection is from left to right) and the other, say at $t_{2}$, is with $\gamma_{\text {aux }} \mid\left[\sigma_{2}\left(r_{0}\right), c_{2}\right]$ where $\zeta_{r}$ enters $\Omega_{\text {aux }}$ again (from right to left). (Remember that $\Omega_{\text {aux }}$ is to the left of $\gamma_{\text {aux }}$.)

(ii) If (32) were not true, then (31) shows that $\zeta_{r} \mid\left[\sigma_{2}(r), \sigma_{2}(r)+1\right]$ would intersect $\partial \Omega_{\mathrm{aux}}$, since $\zeta_{r}\left(\sigma_{2}(r)\right)=\gamma\left(\sigma_{2}(r)\right)$ and $\zeta_{r}\left(\sigma_{2}(r)+1\right)=\gamma\left(\sigma_{1}(r)\right)$.

Altogether, (i) and (ii) show that if (32) were not true, then $\zeta_{r}\left(\left[\sigma_{2}(r)\right.\right.$, $\left.\left.\sigma_{2}(r)+1\right]\right)$ would even contain the whole $\operatorname{arc} \zeta_{r}\left(\left[t_{1}, t_{2}\right]\right)$. However, it is easy to see from our definitions that $\zeta_{r}\left(\left[t_{1}, t_{2}\right]\right)$ and therefore that $\zeta_{r}\left(\left[\sigma_{2}(r), \sigma_{2}(r)+\right.\right.$ $1])$ is more than a half-circle, which contradicts inequality (24). Therefore (32) must be true. Since (31) and (32) show that $\partial \Omega_{r} \subset \bar{\Omega}_{\text {aux }}$ the Jordan curve theorem implies (33).

Let $\left[r_{0}, \bar{r}\right][$ be the maximal interval such that

$$
\gamma\left(\left[\sigma_{1}(r), \sigma_{2}(r)\right]\right) \subset \bar{\Omega}_{\text {aux }} \quad \text { for all } r \in\left[r_{0}, \bar{r}\right][.
$$

(iii) $\left[r_{0}, \bar{r}\right]\left[\right.$ contains $r_{0}$. This is obvious from the definition of $\Omega_{\text {aux }}$.

(iv) $\left[r_{0}, \bar{r}\right]\left[\right.$ is closed. It is obvious from the definition that $\sigma_{1}$ and $\sigma_{2}$ are decreasing and increasing, resp. Now, a straightforward proof shows that $\sigma_{1}$ and $\sigma_{2}$ are left-continuous, i.e. $\sigma_{i}\left(r_{n}\right) \rightarrow \sigma_{i}(r)$ for every sequence $r_{n} \nearrow r$. This implies the assertion at once.

(v) $\left[r_{0}, \bar{r}\right]\left[\right.$ is right-open in $\left[r_{0}, r_{c}\right]$. Let $r_{n} \searrow r \in\left[r_{0}, \bar{r}\right][$ be any sequence in $\left[r_{0}, r_{c}\right]$. If $\bar{r}=r_{c}$ we are done. So let us assume $\bar{r}<r_{c}$. The definition of $\sigma_{i}(i=1,2)$ shows that

$$
\sigma_{1}\left(r_{n}\right) \nearrow \bar{\sigma}_{1} \leq \sigma_{1}(r) \quad \text { and } \quad \sigma_{2}\left(r_{n}\right) \searrow \bar{\sigma}_{2} \geq \sigma_{2}(r) .
$$

If $\bar{\sigma}_{i}=\sigma_{i}(r)$ for $i=1$ or 2 then inequality $(30)(i=1)$ or $(29)(i=2)$, resp. shows that $\dot{\gamma}\left(\bar{\sigma}_{i}\right)$ points outside or inside $\Omega_{\text {aux }}$, resp. Hence, $\gamma\left(\sigma_{i}\left(r_{n}\right)\right) \in \bar{\Omega}_{\text {aux }}$ $(i=1$ or 2 , resp.) for almost all $n$.

If $\bar{\sigma}_{2}>\sigma_{2}(r)$ then the definition of $\sigma_{2}$ shows that $\dot{\gamma}\left(\sigma_{2}(r)\right)$ must be parallel to the tangent of the circle $\operatorname{im} \zeta_{r}$ at $\gamma\left(\sigma_{2}(r)\right)$, i.e. either $\varphi_{2}(r)=0$ or $\varphi_{2}(r)=\pi$. However, inequality (23) shows that only $\varphi_{2}(r)=0$ is possible. 
This implies that $\gamma$ enters $\Omega_{r} \cup \zeta_{r}(] \sigma_{2}(r), \sigma_{2}(r)+1[)$ at $\gamma\left(\sigma_{2}(r)\right)$ and we may even conclude that

$$
\left.\left.\gamma(] \sigma_{2}(r), \bar{\sigma}_{2}\right]\right) \subset \Omega_{r} \cup \zeta_{r}(] \sigma_{2}(r), \sigma_{2}(r)+1[) .
$$

Indeed, $\partial \Omega_{r}=\gamma\left(\left[\sigma_{1}(r), \sigma_{2}(r)\right]\right) \cup \zeta_{r}(] \sigma_{2}(r), \sigma_{2}(r)+1[)$ by definition. Therefore, $\left.\gamma|| \sigma_{2}(r), \bar{\sigma}_{2}\right]$ could leave $\Omega_{r}$ only at some point of $\zeta_{r}(] \sigma_{2}(r), \sigma_{2}(r)+1[)$ since $\gamma$ is injective (see Lemma 10). However, this is impossible since the definition of $\bar{\sigma}_{2}$ implies that $\|\gamma(s)\|_{2} \leq r$ for all $\left.\left.s \in\right] \sigma(r), \bar{\sigma}_{2}\right]$.

A completely analogous proof shows that

$$
\gamma\left(\left[\bar{\sigma}_{1}, \sigma_{1}(r)[) \subset \Omega_{r} \cup \zeta_{r}(] \sigma_{2}(r), \sigma_{2}(r)+1[) \quad \text { if } \bar{\sigma}_{1}<\sigma_{1}(r) .\right.\right.
$$

Now the auxiliary assertion shows that $\gamma\left(\left[\bar{\sigma}_{1}, \sigma_{1}(r)[)\right.\right.$ and $\left.\left.\gamma(] \sigma_{2}(r), \bar{\sigma}_{2}\right]\right)$ are contained in $\Omega_{\text {aux }}$. Therefore, $\gamma\left(\sigma_{i}\left(r_{n}\right)\right) \in \Omega_{\text {aux }}(i=1,2)$ for almost all $n$.

Finally, we conclude from (iii)-(v) that $\left[r_{0}, \bar{r}\right]\left[=\left[r_{0}, r_{c}\right]\right.$, i.e.

$$
\gamma\left(\left[\sigma_{1}(r), \sigma_{2}(r)\right]\right) \subset \bar{\Omega}_{\text {aux }} \quad \text { for all } r \in\left[r_{0}, r_{c}\right] .
$$

Since in addition $\gamma\left(\sigma_{i}\left(r_{c}\right)\right) \in \operatorname{im} \zeta_{r_{c}}(i=1,2)$ and since $\operatorname{im} \zeta_{r_{c}} \cap \bar{\Omega}_{\mathrm{aux}}=$ $\left\{\gamma_{\text {aux }}\left(c_{1}\right)\right\}$ (i.e. it contains exactly one element) we conclude that

$$
\gamma\left(\sigma_{1}\left(r_{c}\right)\right)=\gamma\left(\sigma_{2}\left(r_{c}\right)\right) .
$$

This means that $\gamma$ is not injective - a contradiction to our hypothesis which finally proves the Theorem.

Proof of Theorem 1 (continuation). If $f$ were not injective, Theorem 2 would apply: We take the curve $\gamma$ and the $\varepsilon>0$ as in Theorem 2 and the $s_{1}, s_{2}$ according to Theorem 3 . This yields the estimate

$$
\begin{gathered}
\left(\angle \dot{\gamma}\left(s_{2}\right)-\angle(f \circ \gamma)^{\cdot}\left(s_{2}\right)\right)-\left(\angle \dot{\gamma}\left(s_{1}\right)-\angle(f \circ \gamma)^{\cdot}\left(s_{1}\right)\right) \\
=\angle \dot{\gamma}\left(s_{2}\right)-\angle \dot{\gamma}\left(s_{1}\right)-\left(\angle(f \circ \gamma) \cdot\left(s_{2}\right)-\angle(f \circ \gamma)^{\cdot}\left(s_{1}\right)\right) \\
<\pi+\varepsilon-(3 \pi+\varepsilon)=-2 \pi .
\end{gathered}
$$

Hypothesis 2 of our Theorem 1 shows that

$$
(f \circ \gamma)^{\cdot}=D f(\gamma(s)) \circ \dot{\gamma}(s) \neq \lambda \dot{\gamma}(s) \quad \text { for all } \lambda>0 .
$$

This means that $(f \circ \gamma) \cdot(s)$ and $\dot{\gamma}(s)$ never point in the same direction. Therefore, there must be an open interval of length $2 \pi$, say $] \Psi, \Psi+2 \pi[$, such that $\left.\angle \dot{\gamma}(s)-\angle(f \circ \gamma)^{\cdot}(s) \in\right] \Psi, \Psi+2 \pi[$ for all $s$. However, this obviously contradicts our estimate above. Thus, $f$ must be injective. 


\section{References}

[Ba] N. E. Barabanov, On a problem of Kalman, Siberian Math. J. 29 (3) (1988), 333-341.

[Fe] R. Feßler, A solution of the Global Asymptotic Stability Jacobian Conjecture and a generalization, in: M. Sabatini (ed.), Recent Results on the Global Asymptotic Stability Jacobian Conjecture, Proc. Povo, 1993, Dipartimento di Matematica, Università di Trento.

[GLS] A. Gasull, J. Llibre and J. Sotomayor, Global asymptotic stability of differential equations in the plane, J. Differential Equations 91 (1991), 327-335.

[Gu] C. Gutierrez, A solution of the bidimensional Global Asymptotic Stability Jacobian Conjecture, in: M. Sabatini (ed.), Recent Results on the Global Asymptotic Stability Jacobian Conjecture, Proc. Povo, 1993, Dipartimento di Matematica, Università di Trento.

[Ha] P. Hartman, On stability in the large for systems of ordinary differential equations, Canad. J. Math. 13 (1961), 480-492.

[HO] P. Hartman and C. Olech, On global asymptotic stability of differential equations, Trans. Amer. Math. Soc. 104 (1962), 154-178.

[Kr] N. N. Krasovskiur, Some Problems of the Stability Theory of Motion, Gos. Izdat. Fiz.-Mat. Literat., Moscow, 1959 (in Russian).

[MY] L. Markus and H. Yamabe, Global stability criteria for differential systems, Osaka J. Math. 12 (1960), 305-317.

[MO] G. Meisters and C. Olech, Solution of the global asymptotic stability jacobian conjecture for the polynomial case, in: Analyse Mathématique et Applications, Gauthier-Villars, Paris, 1988, 373-381.

[Ol] C. Olech, On the global stability of an autonomous system on the plane, Contributions to Differential Equations 1 (1963), 389-400.

MATHEMATISCHES INSTITUT DER UNIVERSITÄT BASEL

RHEINSPRUNG 21

CH-4051 BASEL, SWITZERLAND

E-mail: FESSLER@MATH.UNIBAS.CH 